\title{
Regioselective Manipulation of GlcNAc Provides Allosamine, Lividosamine, and Related Compounds
}

\author{
Ji Zhang, Niek N. H. M. Eisink, Martin D. Witte,*두 and Adriaan J. Minnaard*(-) \\ Stratingh Institute for Chemistry, University of Groningen, Nijenborgh 7, 9747 AG Groningen, The Netherlands
}

Supporting Information

ABSTRACT: Palladium-catalyzed oxidation of isopropyl $N$-acetyl- $\alpha$-D-glucosamine (GlcNAc) is used to prepare the rare sugars allosamine, lividosamine, and related compounds with unprecedented selectivity. The Passerini reaction applied on 3-keto-GlcNAc provides an entry into branching of the carbon skeleton in this compound.

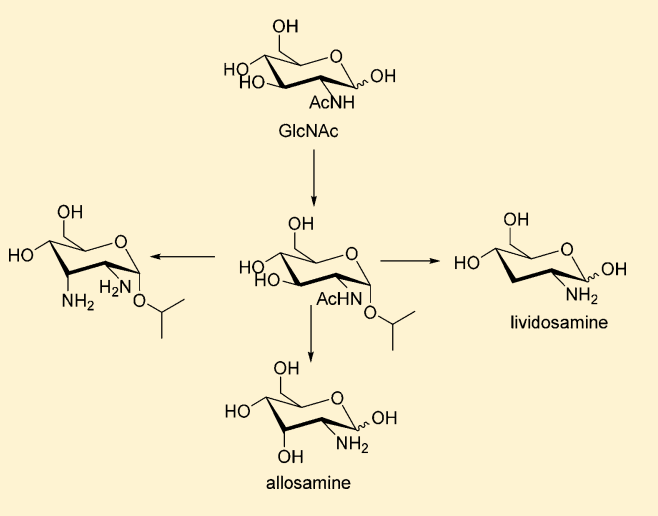

\section{INTRODUCTION}

The hexoses glucose, galactose, mannose, glucosamine, and rhamnose are commonly found in nature. They are part of various $\mathrm{O}$ - and $\mathrm{N}$-glycosylated proteins, glycolipids, and glycans. Besides these hexoses, a large variety of rare sugars have been isolated from natural sources. Altrose, allose, and talose configured monosaccharides have been found in natural products of bacteria in particular. Often these rare monosaccharides are also deoxygenated on one or multiple positions, contain amino groups, and/or have a branched carbon skeleton. ${ }^{1}$ The biological activity of the natural products containing rare sugars necessitates the development of synthesis routes that provide access to these sugars. These less frequently occurring monosaccharides are generally prepared from the readily available hexoses glucose, galactose, mannose, and rhamnose. ${ }^{2}$ This nearly invariably comprises a strategy that protects all-but-one hydroxyl groups, followed by manipulation of the hydroxy group singled out, and finally deprotection. Over the years this approach has reached a high level of sophistication. ${ }^{3-5}$ Inversion of stereocenters has been achieved by converting the singled out hydroxy group into the sulfonate ester and subsequent nucleophilic substitution in $\mathrm{S}_{\mathrm{N}}$ 2-type fashion or by oxidation and subsequent stereoselective reduction. ${ }^{\text {ba-d }}$ Preparation of deoxysugars from protected carbohydrates involves either treatment of the corresponding sulfonate ester ${ }^{7-9}$ (mesylate, tosylate, but preferably triflate) with reactive hydride donors, radical reduction of the corresponding halogen derivative or xanthate, or desulfurization of the corresponding thiosugar.

The protecting group strategy has also been used to convert glucosamine into allosamine; the C3-epimer of glucosamine, and lividosamine, that is C3-deoxy glucosamine. Both aminosugars, even though less frequently encountered in nature than glucosamine, galactosamine, and mannosamine, are certainly relevant. Allosamine forms the core component of the Chitinase inhibitor allosamidin. ${ }^{10}$ Lividosamine is part of the aminoglycosides lividomycin- $\mathrm{A}$ and $-\mathrm{B}$ and is a precursor for the antibiotic thienamycin. ${ }^{11,12}$ As a building block for novel antibiotics and inhibitors, ${ }^{13,14}$ ready access is highly relevant all the more so because allosamine and lividosamine are not commercially available. A downside of the reported routes is that even for these apparently simple transformations, epimerization of the hydroxyl group at $\mathrm{C} 3$ and deoxygenation, the number of reaction steps, often involving purification, is already considerable.

With the current state of homogeneous catalysis, the development and application of so-called site-selective catalysis to prepare less accessible saccharides is an attractive strategy, also to avoid the use of protecting groups. ${ }^{15} \mathrm{We}$ and Waymouth's group have shown that site-selective palladiumcatalyzed oxidation of unprotected carbohydrates, ${ }^{16-21}$ including glucose and $\mathrm{N}$-acetyl glucosamine (Scheme 1), is highly efficient. The formed carbonyl function at $\mathrm{C} 3$ should be amendable to several transformations without the requirement to protect the remaining hydroxyl groups, though not at all a trivial task considering the tendency of the carbonyl group to enolize or form the corresponding hydrate. Nevertheless, we considered this development an opportunity to gain a more efficient access to allosamine and lividosamine as well as related diaminosugars and branched aminosugars that are found in nature, mainly in bacteria.

We present here a route that is significantly more efficient, as it makes protection of the $\mathrm{C} 4$ and $\mathrm{C} 6$ hydroxy groups obsolete.

Received: August 7, 2018

Published: December 20, 2018 
Scheme 1. Site-Selective Palladium-Catalyzed Oxidation of Unprotected Carbohydrates

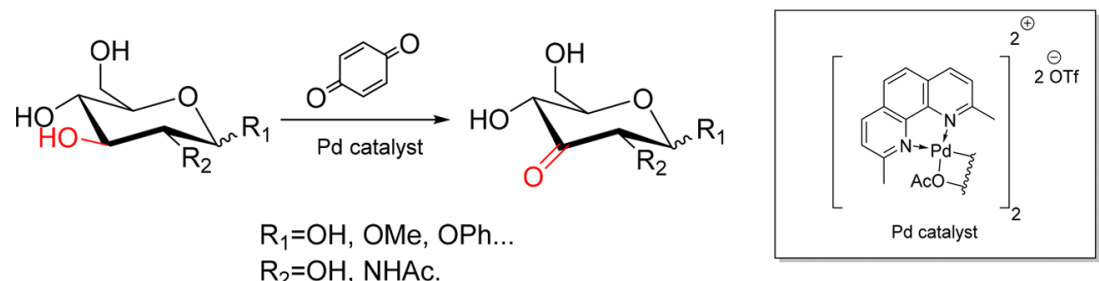

Scheme 2. Jeanloz Synthesis of D-Allosamine and D-N-Acetyl Allosamine

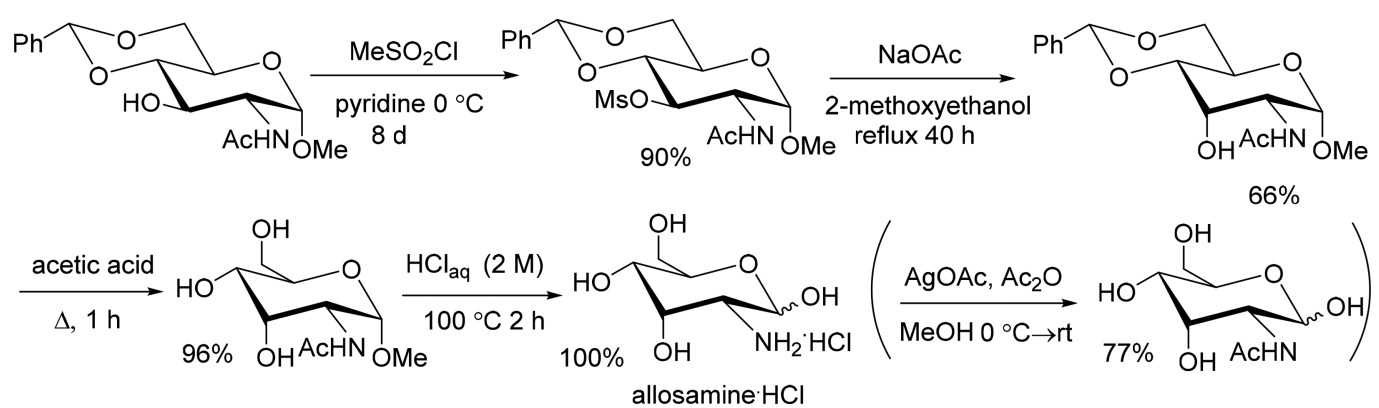

Scheme 3. Synthesis of D-Allosamine and N-Acetyl-D-allosamine

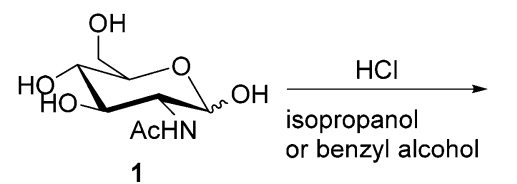

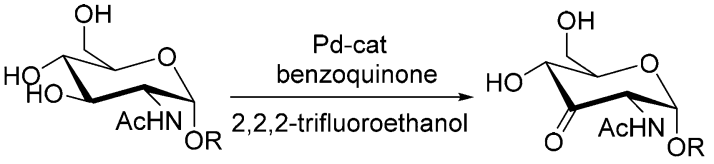

$6 \mathrm{R}=i-\operatorname{Pr}, 77 \%$

$9 \mathrm{R}=\mathrm{Bn}, 58 \%$

$\mathrm{R}=i-\mathrm{Pr}$
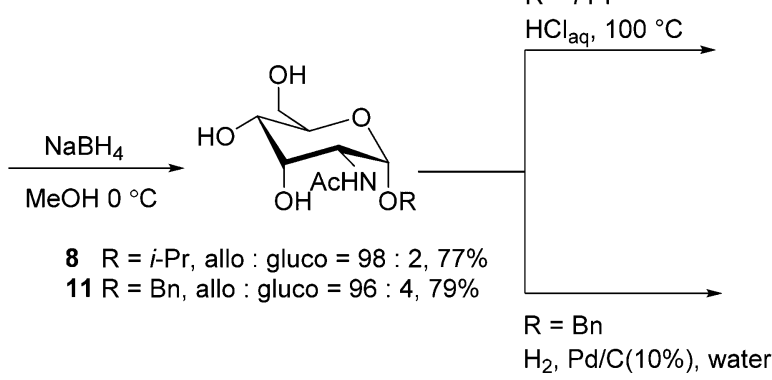

$8 \mathrm{R}=i-\mathrm{Pr}$, allo : gluco $=98: 2,77 \%$

$11 \mathrm{R}=\mathrm{Bn}$, allo $:$ gluco $=96: 4,79 \%$

$\mathrm{R}=\mathrm{Bn}$

$\mathrm{H}_{2}, \mathrm{Pd} / \mathrm{C}(10 \%)$, water
$7 \mathrm{R}=i-\operatorname{Pr}, 89 \%$

$10 \mathrm{R}=\mathrm{Bn}, 91 \%$
$592 \%$

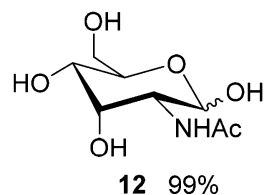

This approach is also used in a more efficient synthesis of lividosamine and an example of the use of unprotected carbohydrates in the Passerini multicomponent reaction.

\section{RESULTS AND DISCUSSION}

To prepare allosamine, Jeanloz et al. inverted the stereocenter at C3 in GlcNAc (Scheme 2). ${ }^{22}$ First GlcNAc was converted into methyl-GlcNAc, and subsequently into its 4,6-benzylidene derivative. Mesylation at $\mathrm{C} 3$ in a slow reaction is then followed by $\mathrm{S}_{\mathrm{N}} 2$-substitution with acetate and hydrolysis to provide methyl 2-acetamido-4,6-O-benzylidene-2-deoxy- $\alpha$-D-allopyranoside. Hydrolysis of the benzylidene group results in methyl $N$-acetyl- $\alpha$-D-allosamine. Finally, hydrolysis with aqueous hydrochloric acid provides allosamine. Alternatively, treatment with silver acetate in acetic anhydride leads to $\mathrm{N}$-acetyl-Dallosamine. Even though this route reported by Jeanloz in 1957 is laborious, it still appears to be the method of choice. The alternative routes to prepare allosamine that have been reported over the years occasionally have comparable or somewhat higher yields, but the step-count is invariably higher also because the required starting materials are not available and therefore have to be prepared. ${ }^{23-25}$

For the synthesis of lividosamine, GlcNAc is deoxygenated at $\mathrm{C} 3$. Arguably the most efficient procedure to lividosamine (2,3-dideoxy-2-aminoglucose) currently is the approach reported by Zhao et al. ${ }^{26}$ GlcNAc is converted into the corresponding isopropylidene protected furanosyl oxazoline, and the C3 hydroxy group is converted into a xanthate, followed by radical deoxygenation with $\mathrm{Bu}_{3} \mathrm{SnH}$, and finally hydrolysis to provide lividosamine. ${ }^{27 a-f}$

We first focused our attention on the synthesis of allosamine by site-selective oxidation followed by stereoselective reduction. Although our palladium-catalyzed oxidation is effective on the parent GlcNAc, ${ }^{19}$ subsequent reduction with $\mathrm{NaBH}_{4}$ is not selective toward $N$-acetyl allosamine, whereas reduction of the corresponding $\alpha$-methyl analogue is. L-Selectride was effective for the stereoselective reduction of 3-ketoglucose, ${ }^{19}$ but subsequent purification was not straightforward. As we desired 

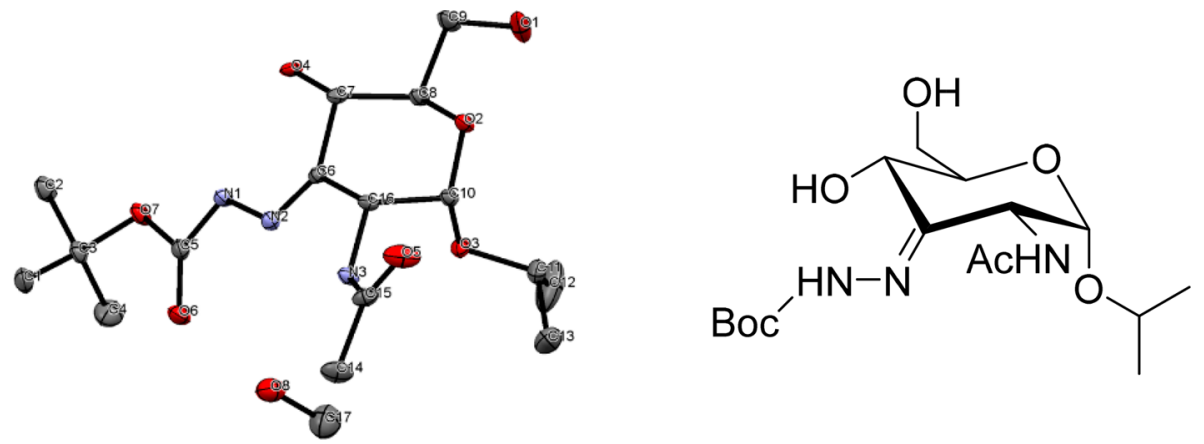

Figure 1. X-ray structure of boc-hydrazone 24 .

Scheme 4. Synthesis of D-Lividosamine

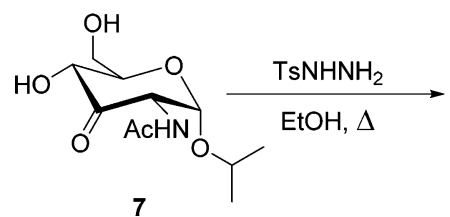

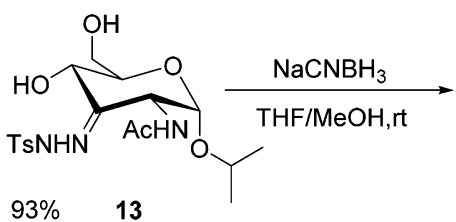
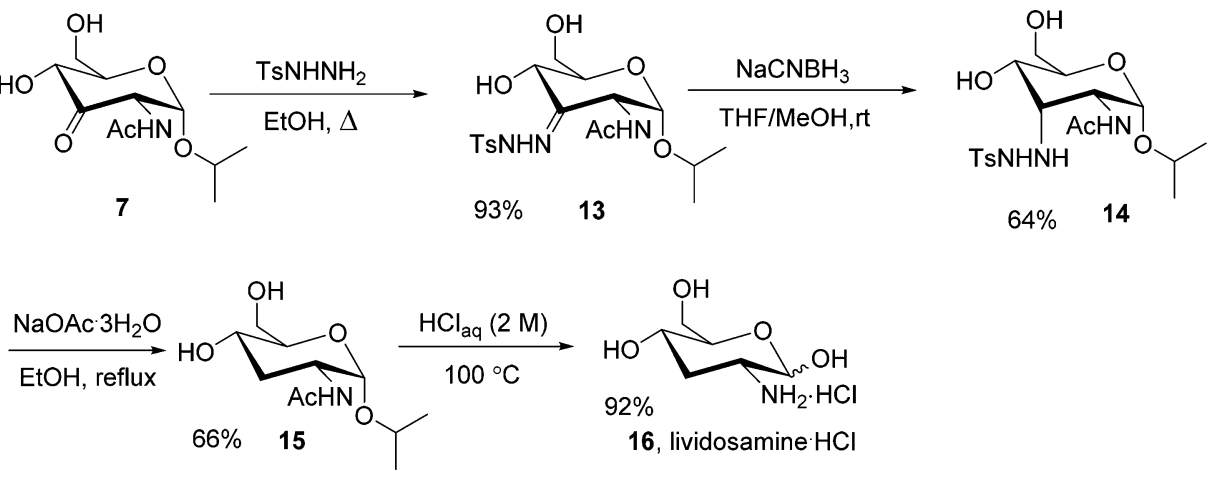

Scheme 5. Synthesis of 2,3-Di-amino Glucose and a Corresponding Fused Imidazole

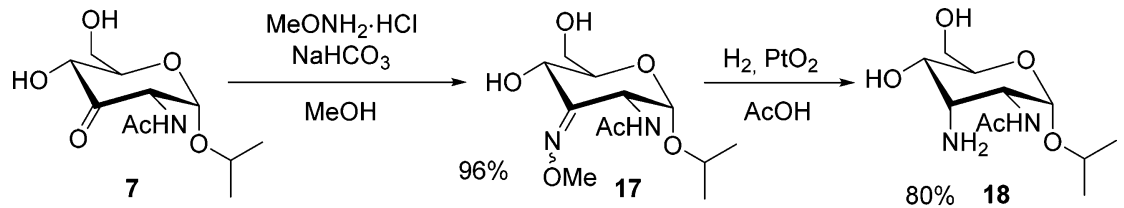

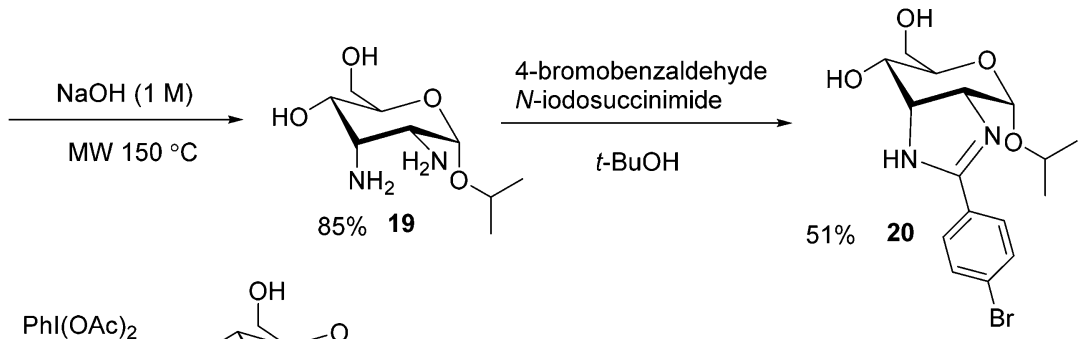<smiles>CCCOc1nc(-c2ccc(Br)cc2)[nH]c1C(O)COc1ccccc1OC(C)C</smiles>

preparative amounts of allosamine, the reduction of 3-ketoGlcNAc with L-selectride was discarded. Attempts to oxidize methyl $\alpha$-D-glucosamine in which the amino group was protected by protonation failed; no reaction was observed.

Fischer glycosylation of GlcNAc with methanol affords an anomeric mixture with a 9.8 to 1 ratio of the $\alpha$ and $\beta$ anomers of 2, respectively (see SI Scheme S1 for compounds 2 and $\mathbf{4}$ and an X-ray structure of 4 ), but removal of the $\beta$-anomer of 2 by column chromatography is difficult. Carrying out the reaction with isopropyl alcohol gave a comparable $\alpha$ to $\beta$ ratio of 9 to 1 , but in this case, the anomeric mixture was readily separated by column chromatography. We observed in a later stage (vide infra) that the reduction of the $\mathrm{C} 3$ carbonyl in the $\alpha$-isopropyl analogue was slightly more stereoselective. Oxidation of the $\beta$-anomer of isopropyl $N$-acetyl-D-glucosamine and subsequent reduction was, as expected, considerably less selective and afforded a 2:1 mixture of the gluco- and alloconfigured products (see SI). This observation made 
Scheme 6. Passerini Reaction with 1-Isopropyl-3-keto GIcNAc
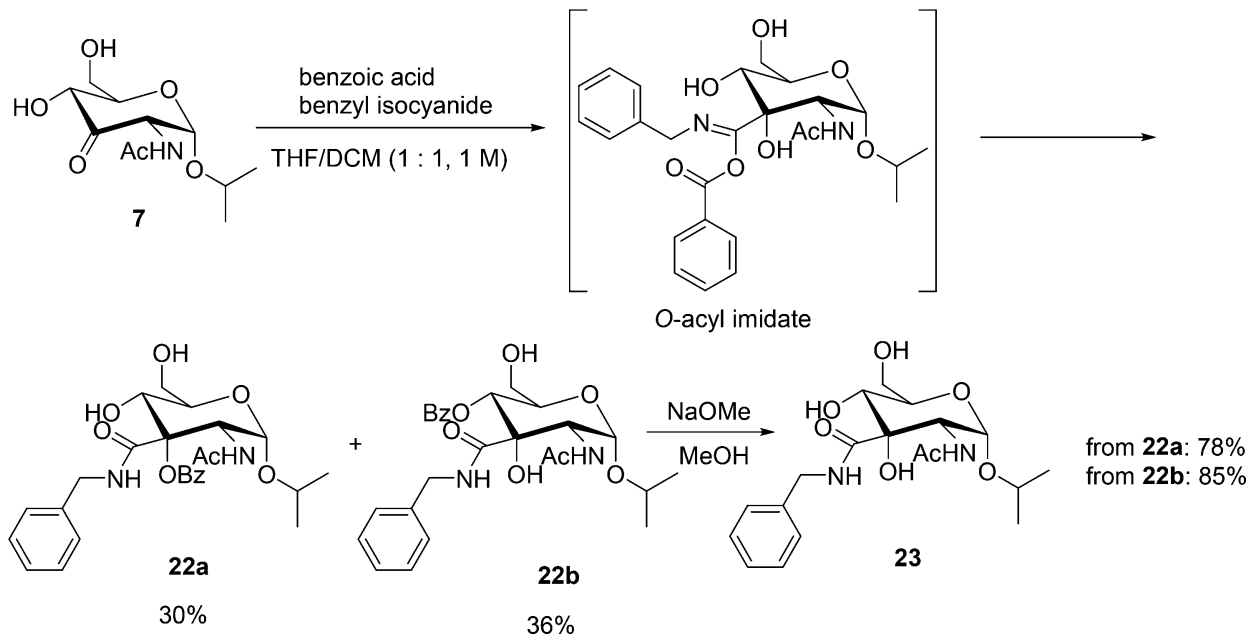

isopropyl- $\alpha$-GlcNAc 6 the starting material of choice (Scheme 3 ). In addition, benzyl- $\alpha$-GlcNAc 9 was prepared as the benzyl substituent and can be removed with mild hydrogenolysis (Scheme 3).

Catalytic oxidation proceeded smoothly to produce ketone 7 in $89 \%$ yield. Its structure was confirmed by X-ray crystal analysis of the corresponding Boc-hydrazone 24 (Figure 1, see also SI). Trifluoroethanol was chosen as the solvent for this reaction, according to Waymouth et al., ${ }^{21}$ as it is more readily removed compared to DMSO. Subsequent $\mathrm{NaBH}_{4}$ reduction provided isopropyl $\mathrm{N}$-acetyl allosamine in a 98 to 2 allo to glucose ratio, the latter being readily removed by column chromatography. Hydrolysis under acidic conditions provided allosamine in $92 \%$ yield. Overall, this route provides pure allosamine in 4 steps, $49 \%$ yield, a significant improvement in yield and stepcount compared to the existing procedures; also compared to the one of Jeanloz, as in that procedure, the starting material requires an additional two steps. When the synthesis was carried out with the benzyl analogue, hydrogenolysis afforded $N$-acetyl allosamine 12 in $41 \%$ overall yield.

We next focused our attention on the synthesis of lividosamine. We reasoned that deoxygenation of the carbonyl function in 7, in the presence of hydroxyl groups, would lead directly to isopropyl $N$-acetyl lividosamine 15 . The number of reactions that converts ketones directly into the corresponding methylene group is limited, and the most appropriate one in the current situation seemed a Caglioti-type reaction, that is, reduction of the corresponding tosylhydrazone. ${ }^{28}$ This reaction, however, had not been applied on unprotected carbohydrates. As expected, synthesis of the tosylhydrazone was uneventful. We were pleased to see that subsequent reduction with $\mathrm{NaCNBH}_{3}$ in methanol and tetrahydrofuran under slightly acidic conditions, followed by elimination with $\mathrm{NaOAc}$ provided 15 (isopropyl 2,3-dideoxy-2- $\mathrm{N}$-acetyl glucosamine). Subsequent hydrolysis provided lividosamine (Scheme 4). Our route to lividosamine is not more efficient than the one of Zhao et al., ${ }^{26}$ but it does avoid the use of tin reagents and applies the same building block as the synthesis of allosamine.

We had shown earlier in the glucose series that reductive amination of the $\mathrm{C} 3$ carbonyl provides an efficient route to 3amino glucose. ${ }^{16}$ Here, we used this strategy on 3-keto GlcNAc 7 as well. Synthesis of the methyl oxime 17 (formed as a 1:1 mixture of $E$ and $Z$ isomers) was followed by hydrogenolysis/ hydrogenation with Adams' catalyst and hydrogen (Scheme 5).
This provided the axially oriented 3-amino group, as expected, because of the shielding by the anomeric isopropyl substituent. After hydrolysis of the acetamide, 2,3-dideoxy-2,3-diaminoallose 19 is obtained. As an illustration that this compound, next to being valuable itself, is a suitable building block for heterocycle synthesis, 19 was condensed with benzaldehyde to provide imidazoline 20. Subsequent oxidation with PIDA provides the corresponding imidazole 21. Remarkably, compounds with this or related scaffolds have hardly been reported ${ }^{29}$ and are therefore a viable addition to the "chemical space" used in medicinal chemistry.

Carbon-carbon bond formation reactions involving unprotected carbohydrates have recently received attention due to the work of Mahrwald et al. ${ }^{30}$ Our group reported on siteselective carbon-carbon bond formation in unprotected monosaccharides at $\mathrm{C} 3$ using photoredox catalysis that allows the formation of branched scaffolds. ${ }^{31}$ Furthermore, we have shown that overoxidation during the palladium-catalyzed oxidation results in branched scaffolds as well. ${ }^{19}$ Also nucleophilic attack of carbon nucleophiles at the carbonyl function in 7 falls in this class. ${ }^{32-34}$ Here we present the use of the multicomponent Passerini reaction in this context. Treatment of 7 with benzyl isocyanide and benzoic acid in THF/DCM (1:1, $1 \mathrm{M})$ provided the expected 3-acyloxy Passerini product 22a. NMR analysis of this product showed that it had the indicated stereochemistry. Presumably, the shielding by the anomeric isopropyl substituent blocks attack from the bottom face and thus prevents the formation of the other epimer. In addition to 22a, we isolated a second product $\mathbf{2 2 b}$, which revealed to be a regioisomer of 22a (Scheme 6). The formation of $\mathbf{2 2 b}$ may be explained by the mechanism of Passerini reaction. During the reaction, a reactive $O$-acyl imidate intermediate is formed. This intermediate acylates a neighboring hydroxyl group. Normally, the newly formed hydroxy group is the only that qualifies for acyl transfer, but in our case, both the $\mathrm{C} 3 \mathrm{OH}$ and the $\mathrm{C} 4 \mathrm{OH}$ are in proximity. Hydrolysis of the product $\mathbf{2 2} \mathbf{a}$ and $\mathbf{2 2 b}$, respectively, provided the same product 23 (Scheme 6).

\section{CONCLUSION}

Site-selective catalytic oxidation of GlcNAc is the key step in novel entries to several rare aminosugars and related building blocks. This study shows that unprotected carbohydrates, in 
the present case GlcNAc, are more amendable to selective modification and conversion than generally assumed and that with a careful selection of reaction conditions, many transformations, in the presence of several free hydroxyl groups, are possible.

\section{EXPERIMENTAL SECTION}

General Information. All solvents used for reaction, extraction, filtration, and chromatography were of commercial grade and used without further purification. [(neocuproine $) \operatorname{Pd}(\mu$-OAc $)]_{2}(\mathrm{OTf})_{2}$ was prepared according to the literature procedure. ${ }^{35}$ Flash chromatography was performed on a Reveleris X2 Flash Chromatography, using Grace Reveleris Silica flash cartridges $(4 \mathrm{~g}, 12 \mathrm{~g}, 15 \mathrm{~g}, 24 \mathrm{~g}, 40 \mathrm{~g}, 80 \mathrm{~g}$, and $120 \mathrm{~g}$ ) and Scorpius Diol (OH) $48 \mathrm{~g} .{ }^{1} \mathrm{H}-,{ }^{13} \mathrm{C}-$-, APT-, HSQC-, and COSY-NMR were recorded on a Varian AMX400 spectrometer $\left(400,100 \mathrm{MHz}\right.$, respectively) using DMSO- $d_{6}, \mathrm{D}_{2} \mathrm{O}$, or methanol- $d_{4}$ as solvent. Chemical shift values are reported in ppm with the solvent resonance as the internal standard (DMSO- $d_{6}: \delta 2.50$ for ${ }^{1} \mathrm{H}, \delta 39.52$ for ${ }^{13} \mathrm{C}, \mathrm{CD}_{3} \mathrm{OD}: \delta 3.31$ for ${ }^{1} \mathrm{H}, \delta 49.15$ for ${ }^{13} \mathrm{C}$; $\mathrm{D}_{2} \mathrm{O}: \delta 4.80$ for ${ }^{1} \mathrm{H}$ ). Data are reported as follows: chemical shifts $(\delta)$, multiplicity ( $\mathrm{s}=$ singlet, $\mathrm{d}=$ doublet, $\mathrm{dd}=$ double doublet, $\mathrm{ddd}=$ double double doublet, $\mathrm{t}=$ triplet, appt $=$ apparent triplet, $\mathrm{q}=$ quartet, $\mathrm{m}=$ multiplet), coupling constants $J(\mathrm{~Hz})$, and integration. Highresolution mass measurements were performed using a ThermoScientific LTQ OribitrapXL spectrometer.

Compound Synthesis and Characterization. Methyl 2Acetamido-2-deoxy- $\alpha$-D-glucopyranoside (2). A suspension of $\mathrm{N}$ acetyl glucosamine $(10 \mathrm{~g}, 0.045 \mathrm{~mol})$ and dry Amberlite IR $120 \mathrm{H}^{+}$ $(12 \mathrm{~g})$ in $\mathrm{MeOH}(300 \mathrm{~mL})$ was heated at reflux for $48 \mathrm{~h}$. Upon cooling, the Amberlite resin was removed by filtration, and the methanol removed in vacuo to provide the product $9.98 \mathrm{~g}$ as a mixture of $\alpha$ and $\beta$, yield: $94 \%$, as a white solid $(\alpha: \beta=9.8: 1)$. Five $\mathrm{g}$ of this mixture was purified by flash chromatography on a $120 \mathrm{~g}$ silica cartridge with $\mathrm{DCM} / \mathrm{MeOH}$, and increasing ratio of $\mathrm{MeOH}$ from 0 to $20 \%$ in $50 \mathrm{~min}$, the product eluted at $14 \% \mathrm{MeOH}$ to afford pure methyl 2-acetamido-2-deoxy-D-glucopyranoside as white solid (2.37 g, yield: $47 \%) \mathrm{mp} 188-189{ }^{\circ} \mathrm{C}$ (lit. $\left.{ }^{36} 186-188{ }^{\circ} \mathrm{C}\right) ;{ }^{1} \mathrm{H}$ NMR (400 $\mathrm{MHz}$, methanol- $\left.d_{4}\right) \delta 4.65(\mathrm{~d}, J=3.5 \mathrm{~Hz}, 1 \mathrm{H}), 3.90(\mathrm{dd}, J=10.7,3.6$ $\mathrm{Hz}, 1 \mathrm{H}), 3.83(\mathrm{dd}, J=11.9,2.4 \mathrm{~Hz}, 1 \mathrm{H}), 3.69(\mathrm{dd}, J=11.9,5.7 \mathrm{~Hz}$, $1 \mathrm{H}), 3.63(\mathrm{dd}, J=10.7,8.7 \mathrm{~Hz}, 1 \mathrm{H}), 3.54$ (ddd, $J=10.0,5.7,2.4 \mathrm{~Hz}$, $1 \mathrm{H}), 3.37(\mathrm{~s}, 3 \mathrm{H}), 3.36-3.32(\mathrm{~m}, 1 \mathrm{H}), 1.98(\mathrm{~s}, 3 \mathrm{H}) ;{ }^{13} \mathrm{C}\left\{{ }^{1} \mathrm{H}\right\} \mathrm{NMR}$ $\left(101 \mathrm{MHz}\right.$, methanol- $\left.d_{4}\right) \delta 173.8,100.0,73.8,73.1,72.5,62.9,55.6$, 55.5, 22.7. HRMS (ESI-TOF) $m / z:[\mathrm{M}+\mathrm{H}]^{+}$and $[\mathrm{M}+\mathrm{Na}]^{+} \mathrm{Calcd}$ for $\mathrm{C}_{9} \mathrm{H}_{18} \mathrm{NO}_{6} 236.1129$ and $\mathrm{C}_{9} \mathrm{H}_{17} \mathrm{NO}_{6} \mathrm{Na}$ 258.0954; found 236.1132 and 258.0953 .

Methyl 2-Acetamido-2-deoxy- $\alpha$-D-glucopyran-3-ulose (3). Methyl 2-acetamido-2-deoxy- $\alpha$-D-glucopyranoside $2(474 \mathrm{mg}, 2 \mathrm{mmol})$ and benzoquinone (324 mg, $3 \mathrm{mmol}$ ) were dissolved in DMSO $(6.6 \mathrm{~mL})$. The catalyst $[\text { (neocuproine)PdOAc }]_{2} \mathrm{OTf}_{2}(57 \mathrm{mg}, 2.5 \mathrm{~mol} \%)$ was added, and the mixture was stirred at room temperature for $1 \mathrm{~h}$. Upon completion of the reaction (according to TLC), water $(70 \mathrm{~mL}$ ) was added, and the mixture was lyophilized to afford the crude product. Subsequent purification by flash chromatography on a $12 \mathrm{~g}$ silica cartridge with $\mathrm{DCM} / \mathrm{MeOH}$, increasing ratio of $\mathrm{MeOH}$ from 0 to $7 \%$ in $21 \mathrm{~min}$, the product eluted at $4 \% \mathrm{MeOH}$ to afford a white solid (346 mg, 74\%), mp 161-162 ${ }^{\circ} \mathrm{C}\left(\right.$ lit. $\left.^{36} 164{ }^{\circ} \mathrm{C}\right) ;{ }^{1} \mathrm{H}$ NMR (400 $\mathrm{MHz}$, Methanol- $\left.d_{4}\right) \delta 5.09(\mathrm{~d}, J=4.1 \mathrm{~Hz}, 1 \mathrm{H}), 4.88(\mathrm{dd}, J=4.1,1.2$ $\mathrm{Hz}, 1 \mathrm{H}), 4.29$ (dd, $J=9.8,1.3 \mathrm{~Hz}, 1 \mathrm{H}), 3.89$ (dd, $J=12.1,2.3 \mathrm{~Hz}$, $1 \mathrm{H}), 3.82(\mathrm{dd}, J=12.1,4.6 \mathrm{~Hz}, 1 \mathrm{H}), 3.73-3.67(\mathrm{~m}, 1 \mathrm{H}), 3.39(\mathrm{~s}$, $3 \mathrm{H}), 2.03(\mathrm{~s}, 3 \mathrm{H}) ;{ }^{13} \mathrm{C}\left\{{ }^{1} \mathrm{H}\right\}$ NMR $\left(101 \mathrm{MHz}\right.$, methanol- $\left.d_{4}\right) \delta 203.8$, 173.7, 102.4, 77.0, 73.8, 62.6, 60.3, 55.8, 22.4; HRMS (ESI-TOF) $\mathrm{m} /$ $z:[\mathrm{M}+\mathrm{H}]^{+}$and $[\mathrm{M}+\mathrm{Na}]^{+}$Calcd for $\mathrm{C}_{9} \mathrm{H}_{16} \mathrm{NO}_{6} 234.0972$ and $\mathrm{C}_{9} \mathrm{H}_{15} \mathrm{NO}_{6} \mathrm{Na} 256.0797$; found 234.0973 and 256.0793 .

Methyl 2-Acetamido-2-deoxy- $\alpha$-D-allopyranoside (4). Methyl 2acetamido-2-deoxy- $\alpha$-D-glucopyran-3-ulose 3 (346 mg, $1.5 \mathrm{mmol}$ ) was dissolved in $\mathrm{MeOH}(12 \mathrm{~mL})$, and the mixture was cooled to $0{ }^{\circ} \mathrm{C}$. $\mathrm{NaBH}_{4}(170 \mathrm{mg}, 4.5 \mathrm{mmol})$ was added, and the mixture stirred for 1 $\mathrm{h}$ at $0{ }^{\circ} \mathrm{C}$. Upon completion of the reaction, Amberlite $120 \mathrm{H}^{+}$was added until $\mathrm{pH} \sim 7$, as indicated by $\mathrm{pH}$ paper to quench remaining
$\mathrm{NaBH}_{4}$. Subsequent filtration and removal of the solvent in vacuo afforded the crude product. This was purified by flash chromatography on a $12 \mathrm{~g}$ silica cartridge with $\mathrm{DCM} / \mathrm{MeOH}$, and increasing ratio of $\mathrm{MeOH}$ from 0 to $20 \%$ in $21 \mathrm{~min}$, the product eluted at $10 \%$ $\mathrm{MeOH}$ to afford a brown oil ( $295 \mathrm{mg}, 85 \%)$; the product elutes as the mixture of methyl 2-acetamido-2-deoxy- $\alpha$-D-allopyranoside and methyl 2-acetamido-2-deoxy- $\alpha$-D-glucopyranoside (96:4), which is difficult to be separated by silica chromatography. ${ }^{1} \mathrm{H}$ NMR (400 $\mathrm{MHz}$, methanol- $\left.d_{4}\right) \delta 4.67(\mathrm{~d}, J=3.9 \mathrm{~Hz}, 1 \mathrm{H}), 4.05(\mathrm{t}, J=3.6 \mathrm{~Hz}$, $1 \mathrm{H}), 3.92(\mathrm{t}, J=3.3 \mathrm{~Hz}, 1 \mathrm{H}), 3.86(\mathrm{dd}, J=11.3,1.7 \mathrm{~Hz}, 1 \mathrm{H}), 3.80-$ $3.69(\mathrm{~m}, 2 \mathrm{H}), 3.53(\mathrm{dd}, J=9.8,3.2 \mathrm{~Hz}, 1 \mathrm{H}), 3.40(\mathrm{~s}, 3 \mathrm{H}), 2.01(\mathrm{~s}$, $3 \mathrm{H}) ;{ }^{13} \mathrm{C}\left\{{ }^{1} \mathrm{H}\right\}$ NMR $\left(101 \mathrm{MHz}\right.$, methanol- $\left.d_{4}\right) \delta 173.1,99.9,71.5$, 69.1, 68.3, 62.9, 56.1, 51.7, 22.7; HRMS (ESI-TOF) $m / z:[\mathrm{M}+\mathrm{H}]^{+}$ and $[\mathrm{M}+\mathrm{Na}]^{+}$Calcd for $\mathrm{C}_{9} \mathrm{H}_{18} \mathrm{NO}_{6} 236.1129$ and $\mathrm{C}_{9} \mathrm{H}_{17} \mathrm{NO}_{6} \mathrm{Na}$ 258.0954; found 236.1130 and 258.0950

D-Allosamine (5). Methyl 2-acetamido-2-deoxy- $\alpha$-D-allopyranoside $4(295 \mathrm{mg}, 1.2 \mathrm{mmol})$ was dissolved in $\mathrm{HCl}_{\mathrm{aq}}(2 \mathrm{M}, 1.5 \mathrm{~mL})$ and heated at $100{ }^{\circ} \mathrm{C}$ for $2 \mathrm{~h}$. Subsequent evaporation of the volatiles provided allosamine $\cdot \mathrm{HCl}(236 \mathrm{mg}, 87 \%)$ as a brown syrup. Spectral data were identical to those obtained by hydrolysis of isopropyl $\mathrm{N}$ acetyl- $\alpha$-D-allosamine.

Isopropyl 2-Acetamido-2-deoxy- $\alpha$-D-glucopyranoside (6). Acetyl chloride $(1.93 \mathrm{~mL}, 27.12 \mathrm{mmol})$ was slowly added to a suspension of $\mathrm{N}$-acetyl-D-glucosamine $(4.0 \mathrm{~g}, 18.08 \mathrm{mmol})$ in isopropyl alcohol $(160$ $\mathrm{mL}$ ) at room temperature. The suspension was subsequently heated to reflux. The solid dissolved gradually. After $2 \mathrm{~h}$, reaction was finished (according to TLC). At room temperature, $\mathrm{NaHCO}_{3}$ was added until $\mathrm{pH} \sim 7$, and the mixture was stirred for $1 \mathrm{~h}$. Upon filtration and evaporation of the solvent, purification was carried out by flash chromatography on a $120 \mathrm{~g}$ silica cartridge with DCM/ $\mathrm{MeOH}$, and increasing ratio of $\mathrm{MeOH}$ from 0 to $15 \%$ in $38 \mathrm{~min}$, the product eluted at $9 \% \mathrm{MeOH}$ to afford a white solid (3.49 g, 77\%), m.p.: $182-184{ }^{\circ} \mathrm{C}$ (lit. $\left.{ }^{37} 187-189{ }^{\circ} \mathrm{C}\right) ;{ }^{1} \mathrm{H}$ NMR (400 MHz, methanol- $\left.d_{4}\right) \delta 4.90(\mathrm{~d}, J=3.7 \mathrm{~Hz}, 1 \mathrm{H}), 3.93-3.76(\mathrm{~m}, 3 \mathrm{H}), 3.71-$ $3.61(\mathrm{~m}, 3 \mathrm{H}), 3.37-3.32(\mathrm{~m}, 1 \mathrm{H}), 1.97(\mathrm{~s}, 3 \mathrm{H}), 1.22(\mathrm{~d}, J=6.2 \mathrm{~Hz}$ $3 \mathrm{H}), 1.12(\mathrm{~d}, J=6.2 \mathrm{~Hz}, 3 \mathrm{H}) ;{ }^{13} \mathrm{C}\left\{{ }^{1} \mathrm{H}\right\}$ NMR $(101 \mathrm{MHz}$, methanol$\left.d_{4}\right) \delta 173.7,96.8,73.9,72.8,72.6,71.2,62.9,55.7,23.8,22.7,21.8$; HRMS (ESI-TOF) $m / z:[\mathrm{M}+\mathrm{H}]^{+}$and $[\mathrm{M}+\mathrm{Na}]^{+}$Calcd for $\mathrm{C}_{11} \mathrm{H}_{22} \mathrm{NO}_{6} 264.1442$ and $\mathrm{C}_{11} \mathrm{H}_{21} \mathrm{NO}_{6} \mathrm{Na} 286.1267$; found 264.1445 and 286.1265.

Isopropyl 2-Acetamido-2-deoxy- $\alpha$-D-glucopyran-3-uloside (7). Isopropyl 2-acetamido-2-deoxy- $\alpha$-D-glucopyranoside 6 (3.33 g, 12.65 $\mathrm{mmol})$ and benzoquinone $(2.05 \mathrm{~g}, 18.97 \mathrm{mmol})$ were dissolved in 2,2,2-trifluoroethanol $(126 \mathrm{~mL})$. The catalyst [(neocuproine) $\operatorname{Pd}(\mu$ $\mathrm{OAc})]_{2}(\mathrm{OTf})_{2}(133 \mathrm{mg}, 1 \mathrm{~mol} \%)$ was added, and the mixture was stirred at $60{ }^{\circ} \mathrm{C}$ for $1 \mathrm{~h}$. Next, the solvent was evaporated, and the crude product was purified by flash chromatography on a $80 \mathrm{~g}$ silica cartridge with pentane/EtOAc, and increasing ratio of EtOAc from 0 to $100 \%$, the product eluted at $88 \%$ of EtOAc to afford a white solid (2.95 g, 89\%), m.p.: $125-126{ }^{\circ} \mathrm{C}$; ${ }^{1} \mathrm{H}$ NMR (400 MHz, methanol- $\left.d_{4}\right)$ $\delta 5.34(\mathrm{~d}, J=4.2 \mathrm{~Hz}, 1 \mathrm{H}), 4.86(\mathrm{dd}, J=4.4,1.3 \mathrm{~Hz}, 1 \mathrm{H}), 4.28(\mathrm{dd}, J$ $=9.0,1.3 \mathrm{~Hz}, 1 \mathrm{H}), 3.93(\mathrm{p}, J=6.2 \mathrm{~Hz}, 1 \mathrm{H}), 3.89-3.79(\mathrm{~m}, 3 \mathrm{H}), 2.03$ (s, $3 \mathrm{H}), 1.19(\mathrm{~d}, J=6.3 \mathrm{~Hz}, 3 \mathrm{H}), 1.13(\mathrm{~d}, J=6.1 \mathrm{~Hz}, 3 \mathrm{H}) ;{ }^{13} \mathrm{C}\left\{{ }^{1} \mathrm{H}\right\}$ NMR $\left(101 \mathrm{MHz}\right.$, methanol- $\left.d_{4}\right) \delta 203.9,173.5,99.5,77.2,73.9,71.8$, 62.7, 60.4, 23.5, 22.4, 21.6; HRMS (ESI-TOF) $\mathrm{m} / z$ : $[\mathrm{M}+\mathrm{H}]^{+}$and $[\mathrm{M}+\mathrm{Na}]^{+}$Calcd for $\mathrm{C}_{11} \mathrm{H}_{20} \mathrm{NO}_{6} 262.1285$ and $\mathrm{C}_{11} \mathrm{H}_{19} \mathrm{NO}_{6} \mathrm{Na}$ 284.1110 ; found: 262.1287 and 284.1106 .

Isopropyl 2-Acetamido-2-deoxy- $\beta$-D-glucopyran-3-uloside (25). This product was prepared as described for the $\alpha$ anomer starting from isopropyl 2 -acetamido-2-deoxy- $\beta$-D-glucopyranoside. ${ }^{1} \mathrm{H}$ NMR $\left(400 \mathrm{MHz}\right.$, methanol- $\left.d_{4}\right) \delta 4.66(\mathrm{~d}, J=8.3 \mathrm{~Hz}, 1 \mathrm{H}), 4.48(\mathrm{~d}, J=8.3$ $\mathrm{Hz}, 1 \mathrm{H}), 4.22(\mathrm{~d}, J=10.1 \mathrm{~Hz}, 1 \mathrm{H}), 4.08-3.99(\mathrm{~m}, 1 \mathrm{H}), 3.94(\mathrm{dd}, J=$ $12.3,2.2 \mathrm{~Hz}, 1 \mathrm{H}), 3.80(\mathrm{dd}, J=12.1,5.0 \mathrm{~Hz}, 1 \mathrm{H}), 3.40-3.34(\mathrm{~m}$, $1 \mathrm{H}), 2.02(\mathrm{~s}, 3 \mathrm{H}), 1.24(\mathrm{~d}, J=6.1 \mathrm{~Hz}, 3 \mathrm{H}), 1.14(\mathrm{~d}, J=6.1 \mathrm{~Hz}, 3 \mathrm{H})$. ${ }^{13} \mathrm{C}\left\{{ }^{1} \mathrm{H}\right\}$ NMR (101 MHz, methanol- $\left.d_{4}\right) \delta$ 204.2, 173.6, 102.4, 78.1, $74.2,73.5,62.9,62.8,23.8,22.6,22.3$. HRMS (ESI-TOF) $\mathrm{m} / z$ : $[\mathrm{M}+$ $\mathrm{Na}]^{+}$Calcd for $\mathrm{C}_{11} \mathrm{H}_{19} \mathrm{NO}_{6} \mathrm{Na} 284.1105$; found 284.1108.

Isopropyl 2-Acetamido-2-deoxy- $\alpha$-D-allopyranoside (8). Isopropyl 2-acetamido-2-deoxy- $\alpha$-D-glucopyran-3-uloside $7(2.0 \mathrm{~g}, \quad 7.66$ $\mathrm{mmol}$ ) was dissolved in $\mathrm{MeOH}(50 \mathrm{~mL})$, and the mixture was 
cooled to $0{ }^{\circ} \mathrm{C} . \mathrm{NaBH}_{4}(434 \mathrm{mg}, 11.48 \mathrm{mmol})$ was added, and the mixture was stirred for $30 \mathrm{~min}$ at $0{ }^{\circ} \mathrm{C}$. Upon completion of the reaction, methanolic $\mathrm{HCl}(2 \mathrm{M})$ was added slowly until $\mathrm{pH} \sim 7$, as indicated by $\mathrm{pH}$ paper to quench remaining $\mathrm{NaBH}_{4}$. The ratio of isopropyl 2-acetamido-2-deoxy- $\alpha$-D-allopyranoside and isopropyl 2acetamido-2-deoxy- $\alpha$-D-glucopyranoside is approximately 98:2. Purification by flash chromatography on a $40 \mathrm{~g}$ silica cartridge with DCM/ $\mathrm{MeOH}$, increasing ratio of $\mathrm{MeOH}$ from 0 to $15 \%$ in $29 \mathrm{~min}$, pure isopropyl 2-acetamido-2-deoxy- $\alpha$-D-allopyranoside eluted at $7 \%$ $\mathrm{MeOH}$ to afford a white semisolid (1.56 g, 77\%); ${ }^{1} \mathrm{H}$ NMR (400 $\mathrm{MHz}$, methanol- $\left.d_{4}\right) \delta 4.94(\mathrm{~d}, J=3.9 \mathrm{~Hz}, 1 \mathrm{H}), 4.02($ app t, $J=3.6 \mathrm{~Hz}$, $1 \mathrm{H}), 3.98-3.91(\mathrm{~m}, 1 \mathrm{H}), 3.89(\mathrm{t}, J=3.3 \mathrm{~Hz}, 1 \mathrm{H}), 3.87-3.81(\mathrm{~m}$, $2 \mathrm{H}), 3.76-3.70(\mathrm{~m}, 1 \mathrm{H}), 3.53(\mathrm{dd}, J=10.0,3.2 \mathrm{~Hz}, 1 \mathrm{H}), 2.02(\mathrm{~s}$, $3 \mathrm{H}), 1.26(\mathrm{~d}, J=6.3 \mathrm{~Hz}, 3 \mathrm{H}), 1.15(\mathrm{~d}, J=6.1 \mathrm{~Hz}, 3 \mathrm{H}) ;{ }^{13} \mathrm{C}\left\{{ }^{1} \mathrm{H}\right\}$ NMR (101 MHz, methanol- $\left.d_{4}\right) \delta 173.2,97.1,72.1,72.0,69.4,68.4$, 62.9, 51.6, 23.8, 22.6, 21.6. HRMS (ESI-TOF) $m / z:[\mathrm{M}+\mathrm{H}]^{+}$and $[\mathrm{M}+\mathrm{Na}]^{+}$Calcd for $\mathrm{C}_{11} \mathrm{H}_{22} \mathrm{NO}_{6} 264.1442$ and $\mathrm{C}_{11} \mathrm{H}_{21} \mathrm{NO}_{6} \mathrm{Na}$ 286.1267; found 264.1443 and 286.1263.

Isopropyl 2-Acetamido-2-deoxy- $\beta$-D-allopyranoside (26). Isopropyl 2 -acetamido-2-deoxy- $\beta$-D-glucopyran-3-uloside (25) was reduced as described for the $\alpha$ anomer. A 1 to 2 mixture of the allo and gluco configured product was obtained. ${ }^{1} \mathrm{H}$ NMR $(400 \mathrm{MHz}$, methanol- $\left.d_{4}\right) \delta 4.75(\mathrm{~d}, J=8.5 \mathrm{~Hz}, 1 \mathrm{H}), 4.02-3.91(\mathrm{~m}, 2 \mathrm{H}), 3.84$ (dd, $J=11.4,2.0 \mathrm{~Hz}, 1 \mathrm{H}), 3.79(\mathrm{dd}, J=8.5,2.9 \mathrm{~Hz}, 1 \mathrm{H}), 3.76-3.66$ $(\mathrm{m}, 1 \mathrm{H}), 3.66(\mathrm{dd}, J=11.3,5.7 \mathrm{~Hz}, 1 \mathrm{H}), 3.51(\mathrm{dd}, J=9.5,3.0 \mathrm{~Hz}$, $1 \mathrm{H}), 1.98(\mathrm{~s}, 3 \mathrm{H}), 1.19(\mathrm{~d}, J=6.2 \mathrm{~Hz}, 3 \mathrm{H}), 1.13(\mathrm{~d}, J=6.1 \mathrm{~Hz}, 3 \mathrm{H})$. ${ }^{13} \mathrm{C}\left\{{ }^{1} \mathrm{H}\right\}$ NMR (101 MHz, Methanol- $\left.d_{4}\right) \delta 173.1,99.1,75.6,73.0$, $71.8,68.9,63.4,55.0,24.0,22.8,22.4$. HRMS (ESI-TOF) $\mathrm{m} / z:[\mathrm{M}+$ $\mathrm{Na}]^{+}$Calcd for $\mathrm{C}_{11} \mathrm{H}_{21} \mathrm{NO}_{6} \mathrm{Na} 286.1261$; found 286.1264.

Isopropyl 2-Acetamido-2-deoxy- $\beta$-D-glucopyranoside (27). ${ }^{1} \mathrm{H}$ NMR $\left(400 \mathrm{MHz}\right.$, methanol- $\left.d_{4}\right) \delta 4.50(\mathrm{~d}, J=8.0 \mathrm{~Hz}, 1 \mathrm{H}), 3.96(\mathrm{p}, J$ $=6.2 \mathrm{~Hz}, 1 \mathrm{H}), 3.87(\mathrm{dd}, J=11.9,2.2 \mathrm{~Hz}, 1 \mathrm{H}), 3.68(\mathrm{dd}, J=11.9,5.6$ $\mathrm{Hz}, 1 \mathrm{H}), 3.59-3.46(\mathrm{~m}, 2 \mathrm{H}), 3.34-3.23(\mathrm{~m}, 3 \mathrm{H}), 1.97(\mathrm{~s}, 3 \mathrm{H}), 1.19$ $(\mathrm{d}, J=6.2 \mathrm{~Hz}, 3 \mathrm{H}), 1.12(\mathrm{~d}, J=6.1 \mathrm{~Hz}, 3 \mathrm{H}) .{ }^{13} \mathrm{C}\left\{{ }^{1} \mathrm{H}\right\}$ NMR $(101$ $\mathrm{MHz}$, methanol- $\left.d_{4}\right) \delta 173.8,101.3,78.0,76.1,73.1,72.3,63.0,57.9$, 23.9, 23.1, 22.4.

$D$-Allosamine (5). Isopropyl 2-acetamido-2-deoxy- $\alpha$-D-allopyranoside 8 ( $1.53 \mathrm{~g}, 5.81 \mathrm{mmol})$ was dissolved in $\mathrm{HCl}$ aq $(2 \mathrm{M}, 7.0 \mathrm{~mL})$ and heated at $100{ }^{\circ} \mathrm{C}$ for $2 \mathrm{~h}$. Subsequent evaporation of the volatiles provided the product $(1.16 \mathrm{~g}, 92 \%)$ as a brown syrup. The product comes as a mixture of pyranose and furanose forms, the major form being the $\beta$-pyranose. The ${ }^{1} \mathrm{H}$ NMR of D-allosamine as reported in the literature ${ }^{38}$ is in $\mathrm{D}_{2} \mathrm{O}$, and we found that the use of methanol- $d_{4}$ gives a much higher quality spectrum. ${ }^{1} \mathrm{H}$ NMR $\left(400 \mathrm{MHz}\right.$, methanol- $\left.d_{4}\right) \delta$ $5.03(\mathrm{~d}, J=8.3 \mathrm{~Hz}, 1 \mathrm{H}), 4.16(\mathrm{t}, J=3.0 \mathrm{~Hz}, 1 \mathrm{H}), 3.85(\mathrm{dd}, J=11.7$, $2.3 \mathrm{~Hz}, 1 \mathrm{H}), 3.80-3.73(\mathrm{~m}, 1 \mathrm{H}), 3.71-3.65(\mathrm{~m}, 1 \mathrm{H}), 3.56(\mathrm{dd}, J=$ 9.8, $2.9 \mathrm{~Hz}, 1 \mathrm{H}), 3.01(\mathrm{dd}, J=8.4,2.9 \mathrm{~Hz}, 1 \mathrm{H}) ;{ }^{13} \mathrm{C}\left\{{ }^{1} \mathrm{H}\right\} \operatorname{NMR}(101$ MHz, Methanol- $d_{4}$ ) $\delta$ 92.6, 75.9, 69.6, 68.5, 63.0, 56.4; HRMS (ESITOF) $m / z:[\mathrm{M}+\mathrm{H}]^{+}$Calcd for $\mathrm{C}_{6} \mathrm{H}_{14} \mathrm{NO}_{5}$ 180.0867; found 180.0868 .

Benzyl 2-Acetamido-2-deoxy- $\alpha$-D-glucopyranoside (9). Acetyl chloride $(1.9 \mathrm{~mL}, 27.1 \mathrm{mmol})$ was slowly added to the suspension of $\mathrm{N}$-acetyl glucosamine $(4.0 \mathrm{~g}, 18.08 \mathrm{mmol})$ in benzyl alcohol (40 $\mathrm{mL}$ ) and stirred at room temperature for $30 \mathrm{~min}$. The mixture was then heated to $95{ }^{\circ} \mathrm{C}$. After $3 \mathrm{~h}$, the reaction mixture was allowed to cool down at room temperature, followed by the addition of anhydrous $\mathrm{Na}_{2} \mathrm{SO}_{4}(257 \mathrm{mg}, 1.81 \mathrm{mmol})$. Subsequently the reaction was heated to $75{ }^{\circ} \mathrm{C}$ for $3 \mathrm{~h}$ before being cooled to room temperature. The resulting brown solution was slowly poured into $\mathrm{Et}_{2} \mathrm{O}(700 \mathrm{~mL})$. The precipitate was recovered by filtration and purified by flash chromatography on a $120 \mathrm{~g}$ silica cartridge with $\mathrm{DCM} / \mathrm{MeOH}$, and increasing the ratio of $\mathrm{MeOH}$ from 0 to $15 \%$ in $38 \mathrm{~min}$, the product eluted at $9 \% \mathrm{MeOH}$ to afford a white solid (3.28 g, 58\%), m.p.: 175$177{ }^{\circ} \mathrm{C}$ (lit. $\left.{ }^{39} 178-180{ }^{\circ} \mathrm{C}\right) ;{ }^{1} \mathrm{H}$ NMR (400 MHz, methanol- $\left.d_{4}\right) \delta$ $7.43-7.23(\mathrm{~m}, 5 \mathrm{H}), 4.86\left(1 \mathrm{H}\right.$, overlap with the peak of $\left.\mathrm{CD}_{3} \mathrm{OD}\right)$, $4.75(\mathrm{~d}, J=12.0 \mathrm{~Hz}, 1 \mathrm{H}), 4.50(\mathrm{~d}, J=12.0 \mathrm{~Hz}, 1 \mathrm{H}), 3.89(\mathrm{dd}, J=$ 10.7, $3.6 \mathrm{~Hz}, 1 \mathrm{H}), 3.87-3.78(\mathrm{~m}, 1 \mathrm{H}), 3.76-3.62(\mathrm{~m}, 3 \mathrm{H}), 3.42-$ $3.32(\mathrm{~m}, 1 \mathrm{H}), 1.95(\mathrm{~s}, 3 \mathrm{H}) ;{ }^{13} \mathrm{C}\left\{{ }^{1} \mathrm{H}\right\}$ NMR $\left(101 \mathrm{MHz}\right.$, methanol- $\left.d_{4}\right)$ $\delta 173.7,139.2,129.5,129.4,129.0,97.6,74.2,72.8,72.6,70.3,62.9$, 55.6, 22.7; HRMS (ESI-TOF) $m / z:[\mathrm{M}+\mathrm{H}]^{+}$and $[\mathrm{M}+\mathrm{Na}]^{+}$Calcd for $\mathrm{C}_{15} \mathrm{H}_{22} \mathrm{NO}_{6} 312.1442$ and $\mathrm{C}_{15} \mathrm{H}_{21} \mathrm{NO}_{6} \mathrm{Na}$ 334.1267; found 312.1446 and 334.1264.

Benzyl 2-Acetamido-2-deoxy- $\alpha$-D-glucopyran-3-uloside (10). Benzyl 2-acetamido-2-deoxy- $\alpha$-D-glucopyranoside $9(1.28 \mathrm{~g}, 4.11$ $\mathrm{mmol}$ ) and benzoquinone $(667 \mathrm{mg}, 6.17 \mathrm{mmol})$ were dissolved in 2,2,2-trifluoroethanol $(41 \mathrm{~mL})$. The catalyst [(2,9-dimethyl-1,10phenanthroline $)-\operatorname{Pd}(\mu$-OAc $)]_{2}(\mathrm{OTf})_{2}(43 \mathrm{mg}, 1 \mathrm{~mol} \%)$ was added, and the mixture was stirred at $60{ }^{\circ} \mathrm{C}$ for $1 \mathrm{~h}$. Subsequently the solvent was evaporated, and the crude was purified by flash chromatography on a $40 \mathrm{~g}$ silica cartridge with pentane/EtOAc, and increasing the ratio of EtOAc from 0 to $100 \%$ in $29 \mathrm{~min}$, the product eluted at $100 \%$ EtOAc to afford a white solid (1.16 g, 91\%), m.p.: $124-126{ }^{\circ} \mathrm{C} ;{ }^{1} \mathrm{H}$ NMR $\left(400 \mathrm{MHz}\right.$, methanol- $\left.d_{4}\right) \delta 7.38-7.24(\mathrm{~m}, 5 \mathrm{H}), 5.27(\mathrm{~d}, J=4.2$ $\mathrm{Hz}, 1 \mathrm{H}), 4.91(\mathrm{dd}, J=4.2,1.3 \mathrm{~Hz}, 1 \mathrm{H}), 4.73(\mathrm{~d}, J=12.0 \mathrm{~Hz}, 1 \mathrm{H})$, $4.55(\mathrm{~d}, J=12.0 \mathrm{~Hz}, 1 \mathrm{H}), 4.31(\mathrm{dd}, J=9.4,1.3 \mathrm{~Hz}, 1 \mathrm{H}), 3.91-3.76$ $(\mathrm{m}, 3 \mathrm{H}), 2.00(\mathrm{~s}, 3 \mathrm{H}) ;{ }^{13} \mathrm{C}\left\{{ }^{1} \mathrm{H}\right\}$ NMR (101 MHz, methanol- $\left.d_{4}\right) \delta$ 203.7, 173.5, 138.4, 129.6, 129.5, 129.2, 100.2, 77.5, 73.9, 70.6, 62.6, 60.3, 22.4; HRMS (ESI-TOF) $\mathrm{m} / z:[\mathrm{M}+\mathrm{H}]^{+}$and $[\mathrm{M}+\mathrm{Na}]^{+} \mathrm{Calcd}$ for $\mathrm{C}_{15} \mathrm{H}_{20} \mathrm{NO}_{6} 310.1285$ and $\mathrm{C}_{15} \mathrm{H}_{19} \mathrm{NO}_{6} \mathrm{Na}$ 332.1110; found 310.1289 and 332.1107.

Benzyl 2-Acetamido-2-deoxy- $\alpha$-D-allopyranoside (11). Benzyl 2acetamide-2-deoxy- $\alpha$-D-glucopyran-3-uloside 10 (935 mg, $3.02 \mathrm{mmol}$ ) was dissolved in $\mathrm{MeOH}(50 \mathrm{~mL})$, and the mixture was cooled to 0 ${ }^{\circ} \mathrm{C} . \mathrm{NaBH}_{4}(172 \mathrm{mg}, 4.53 \mathrm{mmol})$ was added to the mixture, and the mixture was stirred for $30 \mathrm{~min}$ at $0{ }^{\circ} \mathrm{C}$. Upon completion of the reaction, methanolic $\mathrm{HCl}(2 \mathrm{M})$ was added slowly until the $\mathrm{pH}$ reached around 7 (as indicated by $\mathrm{pH}$ paper) to quench remaining $\mathrm{NaBH}_{4}$. The ratio of benzyl 2-acetamido-2-deoxy- $\alpha$-D-allopyranoside and benzyl 2 -acetamido-2-deoxy- $\alpha$-D-glucopyranoside is approximately 96:4. Subsequently, solvents were evaporated, and the crude purified by flash chromatography on a $24 \mathrm{~g}$ silica cartridge with EtOAc/MeOH, and increasing ratio of $\mathrm{MeOH}$ from 0 to $15 \%$ in 21 $\mathrm{min}$, the product eluted at $7 \% \mathrm{MeOH}$ to afford a white solid $(742 \mathrm{mg}$, 79\%), m.p.: $144-145^{\circ} \mathrm{C} ;{ }^{1} \mathrm{H}$ NMR (400 MHz, methanol- $\left.d_{4}\right) \delta 7.43-$ $7.38(\mathrm{~m}, 2 \mathrm{H}), 7.38-7.26(\mathrm{~m}, 3 \mathrm{H}), 4.86$ (overlap with $\mathrm{H}_{2} \mathrm{O}$ in $\left.\mathrm{CD}_{3} \mathrm{OD}, 1 \mathrm{H}\right), 4.78(\mathrm{~d}, J=12.1 \mathrm{~Hz}, 1 \mathrm{H}), 4.54(\mathrm{~d}, J=12.1 \mathrm{~Hz}, 1 \mathrm{H})$, $4.06(\mathrm{t}, J=3.7 \mathrm{~Hz}, 1 \mathrm{H}), 3.92(\mathrm{t}, J=3.3 \mathrm{~Hz}, 1 \mathrm{H}), 3.89-3.82(\mathrm{~m}, 2 \mathrm{H})$, $3.73(\mathrm{dd}, J=12.0,5.7 \mathrm{~Hz}, 1 \mathrm{H}), 3.55(\mathrm{dd}, J=10.2,3.2 \mathrm{~Hz}, 1 \mathrm{H}), 1.98$ $(\mathrm{s}, 3 \mathrm{H}) ;{ }^{13} \mathrm{C}\left\{{ }^{1} \mathrm{H}\right\}$ NMR (101 MHz, methanol- $\left.d_{4}\right) \delta 173.0,139.0$, 129.6, 129.5, 129.1, 97.5, 71.6, 70.7, 69.5, 68.4, 62.9, 51.6, 22.6; HRMS (ESI-TOF) $m / z:[\mathrm{M}+\mathrm{H}]^{+}$and $[\mathrm{M}+\mathrm{Na}]^{+}$Calcd for $\mathrm{C}_{15} \mathrm{H}_{22} \mathrm{NO}_{6} 312.1442$ and $\mathrm{C}_{15} \mathrm{H}_{21} \mathrm{NO}_{6} \mathrm{Na} 334.1267$; found 312.1446 and 334.1265 .

$N$-Acetyl-D-allosamine (12). To a solution of benzyl 2-acetamido2-deoxy- $\alpha$-D-allopyranoside 11 (673 mg, $2.16 \mathrm{mmol})$ in $\mathrm{H}_{2} \mathrm{O}(50 \mathrm{~mL})$ was added $415 \mathrm{mg}$ of $10 \% \mathrm{Pd} / \mathrm{C}$ (supplied by Alfa Aesar, Type 487). The atmosphere was changed to hydrogen (balloon), and the mixture was stirred overnight. The catalyst was removed by filtration, and the filtrate was concentrated to afford the product ( $472 \mathrm{mg}, 99 \%$ ) as a white fluffy solid. The product comes as a mixture of pyranose and furanose forms, the major form being the $\beta$-pyranose. The ${ }^{1} \mathrm{H}$ NMR is consistent with the literature; ${ }^{40}{ }^{1} \mathrm{H}$ NMR $\left(400 \mathrm{MHz}, \mathrm{D}_{2} \mathrm{O}\right) \delta 4.97(\mathrm{~d}$, $J=8.7 \mathrm{~Hz}, 1 \mathrm{H}), 4.11(\mathrm{t}, J=2.9 \mathrm{~Hz}, 1 \mathrm{H}), 3.91(\mathrm{dd}, J=12.1,2.2 \mathrm{~Hz}$, $1 \mathrm{H}), 3.86-3.82(\mathrm{~m}, 1 \mathrm{H}), 3.82-3.78(\mathrm{~m}, 1 \mathrm{H}), 3.75(\mathrm{dd}, J=12.6,6.8$ $\mathrm{Hz}, 1 \mathrm{H}), 3.70(\mathrm{dd}, J=10.1,3.0 \mathrm{~Hz}, 1 \mathrm{H}), 2.07(\mathrm{~s}, 3 \mathrm{H}) ;{ }^{13} \mathrm{C}\left\{{ }^{1} \mathrm{H}\right\} \mathrm{NMR}$ $\left(101 \mathrm{MHz}, \mathrm{D}_{2} \mathrm{O}\right) \delta 174.0,92.3,73.7,69.6,66.4,61.1,54.2,21.8$; HRMS (ESI-TOF) $\mathrm{m} / z$ : $[\mathrm{M}+\mathrm{Na}]^{+}$Calcd for $\mathrm{C}_{8} \mathrm{H}_{15} \mathrm{NO}_{6} \mathrm{Na}$ 244.0792; found: 244.0795 .

$\mathrm{N}$-((2S,3R,5S,6R,Z)-5-Hydroxy-6-(hydroxymethyl)-2-isopropoxy4-(2-tosylhydrazono)tetrahydro-2H-pyran-3-yl)acetamide (13). A mixture of isopropyl 2-acetamide-2-deoxy- $\alpha$-D-ribo-hexapyranoside-3ulose $7(695 \mathrm{mg}, 2.66 \mathrm{mmol})$ and $p$-toluenesulfonyl hydrazide $(743$ $\mathrm{mg}, 3.99 \mathrm{mmol})$ in absolute ethanol $(2.6 \mathrm{~mL})$ was heated at $70{ }^{\circ} \mathrm{C}$ for $3 \mathrm{~h}$ and stirred for $24 \mathrm{~h}$ at room temperature. Then acetic acid (152 $\mu \mathrm{L}, 2.66 \mathrm{mmol}$ ) was added to the reaction mixture. After $5 \mathrm{~h}$, a second portion of acetic acid $(152 \mu \mathrm{L}, 2.66 \mathrm{mmol})$ was added, and the reaction mixture was stirred for another $24 \mathrm{~h}$ until the reaction completed (monitored by TLC). The solvent was evaporated, and the product was purified by flash chromatography on a $24 \mathrm{~g}$ silica cartridge with $\mathrm{DCM} / \mathrm{MeOH}$, and increasing the ratio of $\mathrm{MeOH}$ from 
0 to $4 \%$ in $22 \mathrm{~min}$, the product eluted at $3 \% \mathrm{MeOH}$ to provide a yellow oil (1.06 g, 93\%). ${ }^{1} \mathrm{H}$ NMR (400 MHz, methanol- $\left.d_{4}\right) \delta 7.74$ $(\mathrm{d}, J=8.1 \mathrm{~Hz}, 2 \mathrm{H}), 7.62\left(\mathrm{~d}, J=8.1 \mathrm{~Hz}, 1 \mathrm{H},-\mathrm{SO}_{2} \mathrm{NH}-\right), 7.38(\mathrm{~d}, J=$ $8.0 \mathrm{~Hz}, 2 \mathrm{H}), 5.04(\mathrm{~d}, J=3.6 \mathrm{~Hz}, 1 \mathrm{H}), 4.50-4.43(\mathrm{~m}, 2 \mathrm{H}), 3.84(\mathrm{p}, J$ $=6.2 \mathrm{~Hz}, 1 \mathrm{H}), 3.80-3.74(\mathrm{~m}, 1 \mathrm{H}), 3.73-3.66(\mathrm{~m}, 2 \mathrm{H}), 2.42(\mathrm{~s}, 3 \mathrm{H})$, $2.04(\mathrm{~s}, 3 \mathrm{H}), 1.12(\mathrm{~d}, J=6.3 \mathrm{~Hz}, 3 \mathrm{H}), 1.07(\mathrm{~d}, J=6.1 \mathrm{~Hz}, 3 \mathrm{H})$; ${ }^{13} \mathrm{C}\left\{{ }^{1} \mathrm{H}\right\}$ NMR (101 MHz, methanol- $\left.d_{4}\right) \delta 173.2,173.1,148.8,148.7$, $145.6,137.3,130.9,128.7,97.7,75.7,73.4,71.7,61.9,55.4,23.6,22.6$, 21.8, 21.7; HRMS (ESI-TOF) $m / z:[\mathrm{M}+\mathrm{H}]^{+}$and $[\mathrm{M}+\mathrm{Na}]^{+}$Calcd for $\mathrm{C}_{18} \mathrm{H}_{28} \mathrm{~N}_{3} \mathrm{O}_{7} \mathrm{~S} 430.1643$ and $\mathrm{C}_{18} \mathrm{H}_{27} \mathrm{~N}_{3} \mathrm{O}_{7} \mathrm{SNa}$ 452.1462; found 430.1640 and 452.1454 .

$N-((2 S, 3 R, 4 S, 5 S, 6 R)-5$-Hydroxy-6-(hydroxymethyl)-2-isopropoxy4-(2-tosylhydrazinyl)tetrahydro-2H-pyran-3-yl)acetamide (14). To a stirred solution of the tosylhydrazone $13(822 \mathrm{mg}, 1.9 \mathrm{mmol})$ in a mixture of 1:1 THF-MeOH $(15.2 \mathrm{~mL})$ was added a trace of methyl orange (indicator) and sodium cyanoborohydride $(120 \mathrm{mg}, 1.9$ $\mathrm{mmol})$. Subsequently, methanolic $\mathrm{HCl}(2 \mathrm{M})$ was added dropwise keeping the color of the solution at the red-yellow transition point (orange, $\mathrm{pH} \sim 3.8$ ). The mixture was stirred at room temperature for $1 \mathrm{~h}$. A second portion of sodium cyanoborohydride $(60 \mathrm{mg}, 0.95$ $\mathrm{mmol}$ ) was added, followed by the dropwise addition of methanolic $\mathrm{HCl}(2 \mathrm{M})$ to maintain the $\mathrm{pH}$ at $\sim 3.8$. The mixture was then stirred at room temperature at $\mathrm{pH} \sim 3.8$ for $1 \mathrm{~h}$. $\mathrm{NaHCO}_{3}$ was added to the mixture until $\mathrm{pH} \sim 7$, filtered, and concentrated in vacuo at $40{ }^{\circ} \mathrm{C}$. The residue was purified by flash chromatography on a $24 \mathrm{~g}$ silica cartridge with $\mathrm{DCM} / \mathrm{MeOH}$, and increasing the ratio of $\mathrm{MeOH}$ from $0 \%$ to $4 \%$ in $22 \mathrm{~min}$, the product eluted at $3 \%$ of $\mathrm{MeOH}$ to afford a yellow oil (519 mg, 64\%). ${ }^{1} \mathrm{H}$ NMR $\left(400 \mathrm{MHz}\right.$, methanol- $\left.d_{4}\right) \delta 7.81$ $(\mathrm{d}, J=8.1 \mathrm{~Hz}, 2 \mathrm{H}), 7.47\left(\mathrm{~d}, J=8.5 \mathrm{~Hz}, 1 \mathrm{H},-\mathrm{SO}_{2} \mathrm{NH}-\right), 7.42(\mathrm{~d}, J=$ $8.0 \mathrm{~Hz}, 2 \mathrm{H}), 4.78(\mathrm{~d}, J=3.6 \mathrm{~Hz}, 1 \mathrm{H}), 3.93(\mathrm{dt}, J=8.5,4.3 \mathrm{~Hz}, 1 \mathrm{H})$, $3.87-3.77(\mathrm{~m}, 2 \mathrm{H}), 3.67(\mathrm{dd}, J=11.7,4.5 \mathrm{~Hz}, 1 \mathrm{H}), 3.63-3.56(\mathrm{~m}$, $2 \mathrm{H}), 3.18(\mathrm{t}, J=3.8 \mathrm{~Hz}, 1 \mathrm{H}), 2.44(\mathrm{~s}, 3 \mathrm{H}), 2.03(\mathrm{~s}, 3 \mathrm{H}), 1.06(\mathrm{~d}, J=$ $6.2 \mathrm{~Hz}, 3 \mathrm{H}), 1.04(\mathrm{~d}, J=6.1 \mathrm{~Hz}, 3 \mathrm{H}) ;{ }^{13} \mathrm{C}\left\{{ }^{1} \mathrm{H}\right\} \mathrm{NMR}(101 \mathrm{MHz}$, methanol- $\left.d_{4}\right) \delta 172.8,145.6,137.2,130.9,129.2,96.1,71.2,69.9$, 69.0, 63.7, 62.9, 50.4, 23.8, 22.9, 21.64, 21.59. HRMS (ESI-TOF) $\mathrm{m} /$ $z:\left([\mathrm{M}-\mathrm{H}]^{-}\right.$Calcd for $\mathrm{C}_{18} \mathrm{H}_{28} \mathrm{~N}_{3} \mathrm{O}_{7} \mathrm{~S}$ 430.1643; found: 430.1655 .

Isopropyl 2-Acetamido-2,3-dideoxy- $\alpha$-D-ribo-hexopyranoside (15). A mixture of the tosylhydrazine $14(405 \mathrm{mg}, 0.94 \mathrm{mmol})$ and sodium acetate trihydrate $(511 \mathrm{mg}, 3.75 \mathrm{mmol})$ in $11 \mathrm{~mL}$ of ethanol was refluxed for $3 \mathrm{~h}$. Ethanol was removed in vacuo, and the residue was purified by flash chromatography on a $15 \mathrm{~g}$ silica cartridge with $\mathrm{DCM} / \mathrm{MeOH}$, increasing ratio of $\mathrm{MeOH}$ from 0 to $10 \%$ in $20 \mathrm{~min}$, the product eluted at $5 \% \mathrm{MeOH}$ to provide a white solid $(154 \mathrm{mg}$, 66\%), m.p.: $164-166{ }^{\circ} \mathrm{C}$; ${ }^{1} \mathrm{H}$ NMR (400 MHz, methanol- $\left.d_{4}\right) \delta 4.83$ $(\mathrm{d}, J=3.6 \mathrm{~Hz}, 1 \mathrm{H}), 4.02-3.88(\mathrm{~m}, 2 \mathrm{H}), 3.79(\mathrm{dd}, J=11.7,2.0 \mathrm{~Hz}$, $1 \mathrm{H}), 3.65(\mathrm{dd}, J=11.7,5.0 \mathrm{~Hz}, 1 \mathrm{H}), 3.60-3.49(\mathrm{~m}, 2 \mathrm{H}), 1.94(\mathrm{~s}$, $3 \mathrm{H}), 1.93-1.88(\mathrm{~m}, 1 \mathrm{H}), 1.78(\mathrm{dt}, J=12.7,10.8 \mathrm{~Hz}, 1 \mathrm{H}), 1.25(\mathrm{~d}, J=$ $6.3 \mathrm{~Hz}, 3 \mathrm{H}), 1.13(\mathrm{~d}, J=6.1 \mathrm{~Hz}, 3 \mathrm{H}) ;{ }^{13} \mathrm{C}\left\{{ }^{1} \mathrm{H}\right\}$ NMR $(101 \mathrm{MHz}$, methanol- $\left.d_{4}\right) \delta 172.9,95.3,74.7,70.7,66.4,63.0,49.3,34.0,23.8$, 22.6, 21.9. HRMS (ESI-TOF) $\mathrm{m} / z:[\mathrm{M}+\mathrm{H}]^{+}$and $[\mathrm{M}+\mathrm{Na}]^{+}$Calcd for $\mathrm{C}_{11} \mathrm{H}_{22} \mathrm{NO}_{5} 248.1493$ and $\mathrm{C}_{11} \mathrm{H}_{21} \mathrm{NO}_{5} \mathrm{Na}$ 270.1317; found 248.1494 and 270.1314 .

D-Lividosamine (16). Isopropyl 2 -acetamido-2,3-dideoxy- $\alpha$-D-ribohexopyranoside 15 (143 $\mathrm{mg}, 0.58 \mathrm{mmol}$ ) was dissolved in $\mathrm{HCl}$ (aq) $(2 \mathrm{M}, 0.7 \mathrm{~mL})$ and heated at $100{ }^{\circ} \mathrm{C}$ for $2 \mathrm{~h}$. Subsequent evaporation of the volatiles provided the product ( $107 \mathrm{mg}, 92 \%)$ as a brown syrup. In order to obtain NMR spectra with sharp signals, an analytical sample was dissolved in water, followed by the addition of activated carbon. After filtration and evaporation, the NMR spectra were obtained in DMSO- $d_{6}$. The ${ }^{1} \mathrm{H}$ NMR spectrum shows a major anomeric signal at $\delta 5.12 \mathrm{ppm}$, being the $\alpha$-pyranose of $\mathrm{D}$ lividosamine. $\mathrm{C}_{4}-\mathrm{OH}$ and $\mathrm{C}_{6}-\mathrm{OH}$ are too broad and are difficult to observe in the ${ }^{1} \mathrm{H}$ NMR spectrum. Characterization matches the literature. ${ }^{26}{ }^{1} \mathrm{H}$ NMR (400 MHz, DMSO- $\left.d_{6}\right) \delta 8.18-8.00(\mathrm{~m}, 3 \mathrm{H}$, $\left.\mathrm{C}_{2}-\mathrm{NH}_{2} \mathrm{HCl}\right), 7.12$ (d, J = 4.2 Hz, $\left.1 \mathrm{H}, \mathrm{C}_{1}-\mathrm{OH}\right), 5.12(\mathrm{~d}, J=2.9 \mathrm{~Hz}$, $1 \mathrm{H}, \mathrm{H}$-1 for $\alpha$-isomer), $3.62-3.56(\mathrm{~m}, 1 \mathrm{H}, \mathrm{H}-6 \mathrm{~b}), 3.53-3.44(\mathrm{~m}, 2 \mathrm{H}$, H-5, H-6a), 3.39-3.31 (m, 1H, H-4), 3.15-3.04 (m, 1H, H-2), 2.03$1.96(\mathrm{~m}, 1 \mathrm{H}, \mathrm{H}-3 \mathrm{~b}), 1.65(\mathrm{q}, J=11.8 \mathrm{~Hz}, 1 \mathrm{H}, \mathrm{H}-3 \mathrm{a}) ;{ }^{13} \mathrm{C}\left\{{ }^{1} \mathrm{H}\right\}$ NMR $\left(101 \mathrm{MHz}, \mathrm{DMSO}-d_{6}\right) \delta 87.5,73.2,63.6,60.7,48.3,31.1$. HRMS (ESI-TOF) $m / z:[\mathrm{M}+\mathrm{H}]^{+} \mathrm{C}_{6} \mathrm{H}_{14} \mathrm{NO}_{4}$ 164.0917; found 164.0916.
E/Z-Isopropyl 2-Acetamido-2-deoxy-3-O-methyloxime- $\alpha$-D-ribohexapyranoside (17). Isopropyl 2 -acetamido-2-deoxy- $\alpha$-D-ribo-hexapyranoside-3-uloside 7 (1.57 g, $6.01 \mathrm{mmol})$, methoxyamine hydrochloride $(753 \mathrm{mg}, 9.01 \mathrm{mmol}), \mathrm{NaHCO}_{3}$ (757 mg, 9.01 mmol), and anhydrous $\mathrm{Na}_{2} \mathrm{SO}_{4}(128 \mathrm{mg}, 0.9 \mathrm{mmol})$ were heated in anhydrous methanol $(35 \mathrm{~mL})$ at reflux for $2 \mathrm{~h}$, and subsequently the reaction mixture was stirred at room temperature for 2 days. Evaporation of the solvent provided an oily residue, which was purified by flash chromatography on a $24 \mathrm{~g}$ silica cartridge with $\mathrm{DCM} / \mathrm{MeOH}$, and increasing ratio of $\mathrm{MeOH}$ from 0 to $4 \%$ in $22 \mathrm{~min}$, the product eluted at $3 \%$ to provide an oil $(1.58 \mathrm{~g}, 96 \%$ as a mixture of $E / Z$ isomers). The ratio of $E$ and $Z$ is approximately $1: 1 ;{ }^{1} \mathrm{H}$ NMR (400 MHz, methanol- $d_{4}$ ) Mixture of $E$ and $Z$ isomers: $\delta 5.03$ (d, $J=$ $3.8 \mathrm{~Hz}, 1 \mathrm{H}), 4.95(\mathrm{~d}, J=3.8 \mathrm{~Hz}, 1 \mathrm{H}), 4.83\left(\mathrm{~m}, 1 \mathrm{H}\right.$, overlap with $\mathrm{H}_{2} \mathrm{O}$ in $\left.\mathrm{CD}_{3} \mathrm{OD}\right), 4.65(\mathrm{~d}, J=3.9 \mathrm{~Hz}, 1 \mathrm{H}), 4.45(\mathrm{~d}, J=9.0 \mathrm{~Hz}, 1 \mathrm{H}), 4.10$ $(\mathrm{dt}, J=8.5,3.1 \mathrm{~Hz}, 1 \mathrm{H}), 4.00-3.92(\mathrm{~m}, 2 \mathrm{H}), 3.91-3.86(\mathrm{~m}, 1 \mathrm{H})$, $3.88(\mathrm{~s}, 3 \mathrm{H}), 3.85(\mathrm{~s}, 3 \mathrm{H}), 3.80-3.70(\mathrm{~m}, 5 \mathrm{H}), 2.01(\mathrm{~s}, 3 \mathrm{H}), 1.98(\mathrm{~s}$, $3 \mathrm{H}), 1.24(\mathrm{~d}, J=6.2 \mathrm{~Hz}, 3 \mathrm{H}), 1.23(\mathrm{~d}, J=6.3 \mathrm{~Hz}, 3 \mathrm{H}), 1.16(\mathrm{~d}, J=$ $6.0 \mathrm{~Hz}, 3 \mathrm{H}), 1.15(\mathrm{~d}, J=6.0 \mathrm{~Hz}, 3 \mathrm{H}) ;{ }^{13} \mathrm{C}\left\{{ }^{1} \mathrm{H}\right\}$ NMR $(101 \mathrm{MHz}$, methanol- $\left.d_{4}\right)$ Mixture of $E$ and $Z$ isomers: $\delta$ 173.2, 173.0, 153.2, $151.9,97.5,96.8,77.5,75.4,71.7,71.4,69.6,69.2,63.2,63.1,62.7$, 62.4, 54.3, 53.3, 23.63, 23.58, 22.7, 22.5, 21.8, 21.6. HRMS (ESITOF) $\mathrm{m} / z:[\mathrm{M}+\mathrm{H}]^{+}$and $[\mathrm{M}+\mathrm{Na}]^{+}$Calcd for $\mathrm{C}_{12} \mathrm{H}_{23} \mathrm{~N}_{2} \mathrm{O}_{6}$ 291.1551 and $\mathrm{C}_{12} \mathrm{H}_{22} \mathrm{~N}_{2} \mathrm{O}_{6} \mathrm{Na} 313.1370$; found 291.1562 and 313.1380

Isopropyl 2-Acetamido-3-amino-2,3-dideoxy- $\alpha$-D-allopyranoside (18). E/Z-Isopropyl 2-acetamido-2-deoxy-3-O-methyloxime- $\alpha$-D-ribohexapyranoside $17(1.5 \mathrm{~g}, 5.45 \mathrm{mmol})$ in acetic acid $(26 \mathrm{~mL})$ was hydrogenated over platinum(IV) oxide $(124 \mathrm{mg}, 0.55 \mathrm{mmol}, 10 \mathrm{~mol}$ $\%)$ under hydrogen pressure ( 5 bar) for $24 \mathrm{~h}$. The reaction mixture was filtered over a short path of Celite, and the filtrate was concentrated in vacuo. Purification by Grace flash on a $15 \mathrm{~g}$ silica cartridge with $\mathrm{DCM} / \mathrm{MeOH}$, and increasing ratio of $\mathrm{MeOH}$ from 0 to $20 \%$ in $20 \mathrm{~min}$, the product eluted at $8 \% \mathrm{MeOH}$ to afford a colorless oil $(1.14 \mathrm{~g}, 80 \%)$; ${ }^{1} \mathrm{H}$ NMR $\left(400 \mathrm{MHz}\right.$, methanol- $\left.d_{4}\right) \delta 4.95$ (d, $J=$ $3.6 \mathrm{~Hz}, 1 \mathrm{H}), 4.15(\mathrm{t}, J=3.8 \mathrm{~Hz}, 1 \mathrm{H}), 3.96(\mathrm{p}, J=6.2 \mathrm{~Hz}, 1 \mathrm{H}), 3.87-$ $3.81(\mathrm{~m}, 1 \mathrm{H}), 3.80-3.69(\mathrm{~m}, 3 \mathrm{H}), 3.44(\mathrm{t}, J=4.0 \mathrm{~Hz}, 1 \mathrm{H}), 2.03(\mathrm{~s}$, $3 \mathrm{H}), 1.29(\mathrm{~d}, J=6.2 \mathrm{~Hz}, 3 \mathrm{H}), 1.19(\mathrm{~d}, J=6.1 \mathrm{~Hz}, 3 \mathrm{H}) ;{ }^{13} \mathrm{C}\left\{{ }^{1} \mathrm{H}\right\}$ NMR $\left(101 \mathrm{MHz}\right.$, methanol- $\left.d_{4}\right) \delta 173.4,96.8,72.5,69.3,65.8,62.7$, $54.3,50.2,23.7,22.8,21.7$. HRMS (ESI-TOF) $\mathrm{m} / z:[\mathrm{M}+\mathrm{H}]^{+}$and $[\mathrm{M}+\mathrm{Na}]^{+}$Calcd for $\mathrm{C}_{11} \mathrm{H}_{23} \mathrm{~N}_{2} \mathrm{O}_{5} 263.1602$ and $\mathrm{C}_{11} \mathrm{H}_{22} \mathrm{~N}_{2} \mathrm{O}_{5} \mathrm{Na}$ 285.1426; found 263.1605 and 285.1423;

Isopropyl 2,3-Diamino-2,3-dideoxy- $\alpha$-D-allopyranoside (19). Isopropyl 2-acetamido-3-amino-2,3-dideoxy- $\alpha$-D-allopyranoside 18 (1.07 $\mathrm{g}, 4.08 \mathrm{mmol}$ ) was dissolved in aqueous $\mathrm{NaOH}(1 \mathrm{M}, 8.6 \mathrm{~mL})$. The solution was heated in the microwave for $90 \mathrm{~min}$ at $150{ }^{\circ} \mathrm{C}$ and then cooled down, and the water evaporated. The crude product was purified on a Scorpius Diol $(\mathrm{OH}) 48 \mathrm{~g}$ column using DCM/MeOH, and increasing ratio of $\mathrm{MeOH}$ from 0 to $30 \%$ in $30 \mathrm{~min}$, the product eluted at $5 \% \mathrm{MeOH}$ to afford a yellow oil $(764 \mathrm{mg}, 85 \%) ;{ }^{1} \mathrm{H}$ NMR $\left(400 \mathrm{MHz}\right.$, methanol- $\left.d_{4}\right) \delta 4.82(\mathrm{~d}, J=3.7 \mathrm{~Hz}, 1 \mathrm{H}), 3.92(\mathrm{p}, J=6.2$ $\mathrm{Hz}, 1 \mathrm{H}), 3.83(\mathrm{dd}, J=11.6,2.4 \mathrm{~Hz}, 1 \mathrm{H}), 3.69(\mathrm{dd}, J=11.6,5.5 \mathrm{~Hz}$, $1 \mathrm{H}), 3.65-3.60(\mathrm{~m}, 1 \mathrm{H}), 3.52-3.46(\mathrm{~m}, 1 \mathrm{H}), 3.04(\mathrm{t}, J=4.1 \mathrm{~Hz}$, $1 \mathrm{H}), 2.85-2.80(\mathrm{~m}, 1 \mathrm{H}), 1.25(\mathrm{~d}, J=6.3 \mathrm{~Hz}, 3 \mathrm{H}), 1.17(\mathrm{~d}, J=6.1$ $\mathrm{Hz}, 3 \mathrm{H}) ;{ }^{13} \mathrm{C}\left\{{ }^{1} \mathrm{H}\right\}$ NMR $\left(101 \mathrm{MHz}\right.$, methanol- $\left.d_{4}\right) \delta 99.8,71.8,68.9$, 68.4, 63.2, 56.4, 52.7, 24.1, 21.9; HRMS (ESI-TOF) $m / z:[\mathrm{M}+\mathrm{H}]^{+}$ and $[\mathrm{M}+\mathrm{Na}]^{+}$Calcd for $\mathrm{C}_{9} \mathrm{H}_{21} \mathrm{~N}_{2} \mathrm{O}_{4} 221.1496$ and $\mathrm{C}_{9} \mathrm{H}_{20} \mathrm{~N}_{2} \mathrm{NaO}_{4}$ 243.1321; found 221.1495 and 243.1314 .

(3aR,4S,6R,7S,7aS)-2-(4-Bromophenyl)-6-(hydroxymethyl)-4-isopropoxy-3,3a,4,6,7,7a-hexahydropyrano[3,4-d]imidazol-7-ol (20). A solution of 4-bromobenzaldehyde $(184 \mathrm{mg}, 0.996 \mathrm{mmol})$ in tertbutyl alcohol $(10.9 \mathrm{~mL})$ and isopropyl 2,3-diamino-2,3-dideoxy- $\alpha$-Dallopyranoside $19(241 \mathrm{mg}, 1.094 \mathrm{mmol})$ were mixed and stirred at room temperature for overnight. Subsequently, $\mathrm{N}$-iodosuccinimide ( $246 \mathrm{mg}, 1.09 \mathrm{mmol}$ ) was added to the mixture at room temperature and stirred for $2 \mathrm{~h}$. Sat. aq $\mathrm{NaHCO}_{3}$ was added to the reaction mixture. The mixture was extracted with $\mathrm{CHCl}_{3}$. The organic layer was dried over $\mathrm{MgSO}_{4}$ and evaporated in vacuo. The residue was purified by flash chromatography on a $15 \mathrm{~g}$ silica cartridge with DCM (DCM contains $\left.0.25 \% \mathrm{Et}_{3} \mathrm{~N}\right) / \mathrm{MeOH}$, and increasing the ratio of 
$\mathrm{MeOH}$ from 0 to $5 \%$ in $22 \mathrm{~min}$, the product eluted at $4 \% \mathrm{MeOH}$ to provide a yellow crystalline solid $(216 \mathrm{mg}, 51 \%), \mathrm{mp} 178-182{ }^{\circ} \mathrm{C} ;{ }^{1} \mathrm{H}$ NMR $\left(400 \mathrm{MHz}\right.$, Methanol- $\left.d_{4}\right) \delta 7.79(\mathrm{~s}, 4 \mathrm{H}), 5.08(\mathrm{~d}, J=4.2 \mathrm{~Hz}$, $1 \mathrm{H}), 4.64(\mathrm{dd}, J=9.8,4.8 \mathrm{~Hz}, 1 \mathrm{H}), 4.44(\mathrm{dd}, J=9.8,4.2 \mathrm{~Hz}, 1 \mathrm{H})$, 4.12 (dd, $J=9.5,4.9 \mathrm{~Hz}, 1 \mathrm{H}), 4.00(\mathrm{p}, J=6.2 \mathrm{~Hz}, 1 \mathrm{H}), 3.88-3.75$ $(\mathrm{m}, 3 \mathrm{H}), 1.16(\mathrm{~d}, J=6.2 \mathrm{~Hz}, 3 \mathrm{H}), 1.11(\mathrm{~d}, J=6.1 \mathrm{~Hz}, 3 \mathrm{H}) ;{ }^{13} \mathrm{C}\left\{{ }^{1} \mathrm{H}\right\}$ NMR (101 MHz, methanol- $\left.d_{4}\right) \delta 168.9,133.8,131.2,129.9,124.9$, 94.0, 72.3, 71.8, 63.6, 63.4, 62.9, 60.9, 23.8, 21.9. HRMS (ESI-TOF) $m / z:[\mathrm{M}+\mathrm{H}]^{+}$Calcd for $\mathrm{C}_{16} \mathrm{H}_{22} \mathrm{BrN}_{2} \mathrm{O}_{4} 385.0758$ and 387.0737; found 385.0760 and 387.0734 .

(4S,6R,7S)-2-(4-Bromophenyl)-6-(hydroxymethyl)-4-isopropoxy3,4,6,7-tetrahydropyrano [3, 4-d] imidazol-7-ol (21). To a mixture of $20(193 \mathrm{mg}, 0.5 \mathrm{mmol})$ and $\mathrm{K}_{2} \mathrm{CO}_{3}(76 \mathrm{mg}, 0.55 \mathrm{mmol})$ in DMSO $(5 \mathrm{~mL})$ was added $\mathrm{PhI}(\mathrm{OAc})_{2}(177 \mathrm{mg}, 0.55 \mathrm{mmol})$. Then the mixture was stirred for $24 \mathrm{~h}$ at room temperature under an $\mathrm{N}_{2}$ atomosphere. After the reaction completed, water $(50 \mathrm{~mL})$ was added, and the mixture was lyophilized to afford the crude product. Subsequent purification by flash chromatography on a $4 \mathrm{~g}$ silica cartridge with pentane/EtOAc, and increasing ratio of EtOAc from 0 to $90 \%$ in $15 \mathrm{~min}$, the product eluted at $88 \%$ EtOAc to provide a white amorphous solid (81 mg, 42\%); ${ }^{1} \mathrm{H}$ NMR (400 MHz, methanol- $\left.d_{4}\right) \delta 7.80(\mathrm{~d}, J=8.3 \mathrm{~Hz}, 2 \mathrm{H}), 7.60(\mathrm{~d}, J=8.3 \mathrm{~Hz}, 2 \mathrm{H})$, $5.71(\mathrm{~s}, 1 \mathrm{H}), 4.69(\mathrm{~d}, J=9.0 \mathrm{~Hz}, 1 \mathrm{H}), 4.20(\mathrm{p}, J=6.2 \mathrm{~Hz}, 1 \mathrm{H}), 4.01-$ $3.91(\mathrm{~m}, 2 \mathrm{H}), 3.82(\mathrm{dd}, J=11.9,5.6 \mathrm{~Hz}, 1 \mathrm{H}), 1.30(\mathrm{~d}, J=6.0 \mathrm{~Hz}$, $3 \mathrm{H}), 1.29(\mathrm{~d}, J=6.0 \mathrm{~Hz}, 3 \mathrm{H}) ;{ }^{13} \mathrm{C}\left\{{ }^{1} \mathrm{H}\right\}$ NMR $(101 \mathrm{MHz}$, methanol$\left.d_{4}\right) \delta 147.6,133.2,130.7,128.5,124.0,94.3,75.7,71.6,63.8,62.5$, 24.2, 22.4; HRMS (ESI-TOF) $m / z$ : [M-H] $]^{-}$Calcd for $\mathrm{C}_{16} \mathrm{H}_{18} \mathrm{BrN}_{2} \mathrm{O}_{4}$ 381.0445 and 383.0424; found 381.0452 and 383.0431 .

(2S,3R,4S, 5R,6R)-3-Acetamido-4-(benzylcarbamoyl)-5-hydroxy6-(hydroxymethyl)-2-isopropoxytetrahydro-2H-pyran-4-yl benzoate and (2R,3R,4S,5R,6S)-5-Acetamido-4-(benzylcarbamoyl)-4hydroxy-2-(hydroxymethyl)-6-isopropoxytetrahydro-2H-pyran-3-yl benzoate (22a and 22b). To a stirred suspension of isopropyl 2acetamido-2-deoxy- $\alpha$-D-ribo- hexapyranoside-3-uloside 7 (130 mg, 0.5 $\mathrm{mmol})$ in DCM/THF $(1: 1,0.5 \mathrm{~mL}, 1 \mathrm{M})$ were added benzoic acid $(61 \mathrm{mg}, 0.5 \mathrm{mmol})$ and benzyl isocyanide $(61 \mu \mathrm{L}, 0.5 \mathrm{mmol})$. The reaction was allowed to stir at room temperature for 5 days, then concentrated in vacuo, and separated by flash chromatography on a $12 \mathrm{~g}$ silica cartridge with pentane/EtOAc, and increasing ratio of EtOAc from 0 to $100 \%, 22 \mathrm{a}$ eluted at $77 \%$ EtOAc as colorless oil (74 $\mathrm{mg}, 30 \%)$ and $\mathbf{2 2 b}$ eluted at $90 \%$ EtOAc as white amorphous solid (89 mg, 36\%).

22a. ${ }^{1} \mathrm{H}$ NMR (400 MHz, methanol- $\left.d_{4}\right) \delta 8.01-7.97(\mathrm{~m}, 2 \mathrm{H})$, 7.64-7.58 (m, 1H), 7.50-7.41 (m, 4H), 7.38-7.33 (m, 2H), 7.31$7.24(\mathrm{~m}, 1 \mathrm{H}), 4.99(\mathrm{~d}, J=3.6 \mathrm{~Hz}, 1 \mathrm{H}), 4.82(\mathrm{~d}, J=3.6 \mathrm{~Hz}, 1 \mathrm{H}), 4.65$ $(\mathrm{d}, J=14.9 \mathrm{~Hz}, 1 \mathrm{H}), 4.35(\mathrm{~d}, J=14.9 \mathrm{~Hz}, 1 \mathrm{H}), 4.32(\mathrm{~d}, J=10.1 \mathrm{~Hz}$, $1 \mathrm{H}), 4.14$ (ddd, $J=10.1,4.7,2.5 \mathrm{~Hz}, 1 \mathrm{H}), 3.92(\mathrm{p}, J=6.2 \mathrm{~Hz}, 1 \mathrm{H})$, $3.85(\mathrm{dd}, J=12.0,2.6 \mathrm{~Hz}, 1 \mathrm{H}), 3.79(\mathrm{dd}, J=11.9,4.7 \mathrm{~Hz}, 1 \mathrm{H}), 1.97$ $(\mathrm{s}, 3 \mathrm{H}), 1.13(\mathrm{~d}, J=6.2 \mathrm{~Hz}, 3 \mathrm{H}), 1.10(\mathrm{~d}, J=6.0 \mathrm{~Hz}, 3 \mathrm{H}) ;{ }^{13} \mathrm{C}\left\{{ }^{1} \mathrm{H}\right\}$ NMR $\left(101 \mathrm{MHz}\right.$, methanol- $\left.d_{4}\right) \delta 173.2,169.9,167.2,139.4,134.7$, $131.6,131.1,129.8,129.7,129.1,128.6,96.9,84.5,73.4,71.9,71.9$, 62.7, 55.6, 44.9, 23.6, 23.1, 21.7; HRMS (ESI-TOF) $m / z:[\mathrm{M}+\mathrm{H}]^{+}$ and $[\mathrm{M}+\mathrm{Na}]^{+}$Calcd for $\mathrm{C}_{26} \mathrm{H}_{33} \mathrm{~N}_{2} \mathrm{O}_{8} 501.2231$ and $\mathrm{C}_{26} \mathrm{H}_{32} \mathrm{~N}_{2} \mathrm{O}_{8} \mathrm{Na}$ 523.2056; found 501.2235 and 523.2054;

22b. ${ }^{1} \mathrm{H}$ NMR (400 MHz, methanol- $\left.d_{4}\right) \delta 8.03(\mathrm{~d}, J=7.6 \mathrm{~Hz}, 2 \mathrm{H})$, $7.64(\mathrm{t}, J=7.5 \mathrm{~Hz}, 1 \mathrm{H}), 7.47(\mathrm{t}, J=7.7 \mathrm{~Hz}, 2 \mathrm{H}), 7.07-7.02(\mathrm{~m}, 3 \mathrm{H})$, $7.00-6.94(\mathrm{~m}, 2 \mathrm{H}), 5.57(\mathrm{~d}, J=10.5 \mathrm{~Hz}, 1 \mathrm{H}), 5.08(\mathrm{~d}, J=3.9 \mathrm{~Hz}$, $1 \mathrm{H}), 4.58(\mathrm{~d}, J=3.9 \mathrm{~Hz}, 1 \mathrm{H}), 4.28(\mathrm{~d}, J=15.0 \mathrm{~Hz}, 1 \mathrm{H}), 4.23(\mathrm{~d}, J=$ $14.7 \mathrm{~Hz}, 1 \mathrm{H}), 4.19$ (ddd, $J=10.5,5.1,3.5 \mathrm{~Hz}, 1 \mathrm{H}), 4.03(\mathrm{p}, J=6.2$ $\mathrm{Hz}, 1 \mathrm{H}), 3.68-3.59(\mathrm{~m}, 2 \mathrm{H}), 1.91(\mathrm{~s}, 3 \mathrm{H}), 1.33(\mathrm{~d}, J=6.2 \mathrm{~Hz}, 3 \mathrm{H})$, $1.19(\mathrm{~d}, J=6.1 \mathrm{~Hz}, 3 \mathrm{H}) ;{ }^{13} \mathrm{C}\left\{{ }^{1} \mathrm{H}\right\}$ NMR $\left(101 \mathrm{MHz}\right.$, methanol- $\left.d_{4}\right) \delta$ 173.0, 171.9, 166.6, 139.8, 134.8, 131.2, 130.9, 129.8, 129.4, 128.4, 128.1, 97.1, 79.6, 72.9, 71.4, 68.9, 62.9, 53.0, 44.1, 23.7, 22.5, 21.7; HRMS (ESI-TOF) $m / z:[\mathrm{M}+\mathrm{H}]^{+}$and $[\mathrm{M}+\mathrm{Na}]^{+}$Calcd for $\mathrm{C}_{26} \mathrm{H}_{33} \mathrm{~N}_{2} \mathrm{O}_{8} 501.2231$ and $\mathrm{C}_{26} \mathrm{H}_{32} \mathrm{~N}_{2} \mathrm{O}_{8} \mathrm{Na}$ 523.2056; found 501.2226 and 523.2042 .

(2S, 3R, 4S, 5R,6R)-3-Acetamido-N-benzyl-4,5-dihydroxy-6-(hydroxymethyl)-2-isopropoxytetrahydro-2H-pyran-4-carboxamide (23). To a solution of 22 in methanol $(0.05 \mathrm{M})$ was added sodium methoxide (1.2 equiv) at room temperature. The reaction was stirred at room temperature for $3 \mathrm{~h}$, then concentrated in vacuo, and purified by flash chromatography on a $4 \mathrm{~g}$ silica cartridge with $\mathrm{DCM} / \mathrm{MeOH}$, and increasing ratio of $\mathrm{MeOH}$ from 0 to $15 \%$, the product eluted at $10 \% \mathrm{MeOH}$ to afford a colorless oil.

Obtained 23 from 22a on a $0.116 \mathrm{mmol}$ scale; yield: $36 \mathrm{mg}(78 \%)$. Obtained 23 from $22 \mathbf{b}$ on a $0.136 \mathrm{mmol}$ scale; yield: $46 \mathrm{mg}$ (85\%). HRMS (ESI-TOF) $m / z$ : $[\mathrm{M}-\mathrm{H}]^{-}$Calcd for $\mathrm{C}_{19} \mathrm{H}_{27} \mathrm{~N}_{2} \mathrm{O}_{7} 395.1813$; found 395.1825. ${ }^{1} \mathrm{H}$ NMR (400 MHz, methanol- $\left.d_{4}\right) \delta 7.41-7.24(\mathrm{~m}$, $5 \mathrm{H}), 4.94(\mathrm{~d}, J=4.4 \mathrm{~Hz}, 1 \mathrm{H}), 4.61(\mathrm{~d}, J=14.7 \mathrm{~Hz}, 1 \mathrm{H}), 4.33(\mathrm{~d}, J=$ $14.7 \mathrm{~Hz}, 1 \mathrm{H}), 4.26(\mathrm{~d}, J=4.4 \mathrm{~Hz}, 1 \mathrm{H}), 4.02$ (ddd, $J=10.4,5.3,2.5$ $\mathrm{Hz}, 1 \mathrm{H}), 3.90(\mathrm{p}, J=6.2 \mathrm{~Hz}, 1 \mathrm{H}), 3.82(\mathrm{dd}, J=11.9,2.5 \mathrm{~Hz}, 1 \mathrm{H})$, $3.72(\mathrm{dd}, J=11.9,5.3 \mathrm{~Hz}, 1 \mathrm{H}), 3.66(\mathrm{~d}, J=10.4 \mathrm{~Hz}, 1 \mathrm{H}), 1.96(\mathrm{~s}$, $3 \mathrm{H}), 1.04(\mathrm{~d}, J=6.1 \mathrm{~Hz}, 3 \mathrm{H}), 1.02(\mathrm{~d}, J=6.2 \mathrm{~Hz}, 3 \mathrm{H}) ;{ }^{13} \mathrm{C}\left\{{ }^{1} \mathrm{H}\right\}$ NMR $\left(101 \mathrm{MHz}\right.$, methanol- $\left.d_{4}\right) \delta 173.8,172.3,139.7,129.8,129.2$, 128.6, 96.4, 78.9, 74.5, 72.5, 72.1, 63.0, 56.8, 44.7, 23.3, 22.8, 21.6.

tert-Butyl (Z)-2-((2S,3R,5S,6R)-3-Acetamido-5-hydroxy-6-(hydroxymethyl)-2-isopropoxytetrahydro-4H-pyran-4-ylidene)hydrazine-1-carboxylate (24). ${ }^{1} \mathrm{H}$ NMR $\left(400 \mathrm{MHz}\right.$, methanol- $\left.d_{4}\right) \delta$ $8.36(\mathrm{~d}, J=7.0 \mathrm{~Hz}, 1 \mathrm{H}), 5.20(\mathrm{~d}, J=3.5 \mathrm{~Hz}, 1 \mathrm{H}), 4.56(\mathrm{~d}, J=9.8 \mathrm{~Hz}$, $1 \mathrm{H}), 4.48(\mathrm{dd}, J=7.0,3.5 \mathrm{~Hz}, 1 \mathrm{H}), 3.91-3.85(\mathrm{~m}, 2 \mathrm{H}), 3.80-3.72$ (m, $2 \mathrm{H}), 2.04(\mathrm{~s}, 3 \mathrm{H}), 1.51(\mathrm{~s}, 9 \mathrm{H}), 1.19(\mathrm{~d}, J=6.3 \mathrm{~Hz}, 3 \mathrm{H}), 1.10(\mathrm{~d}$, $J=6.1 \mathrm{~Hz}, 3 \mathrm{H}) \cdot{ }^{13} \mathrm{C}\left\{{ }^{1} \mathrm{H}\right\}$ NMR $\left(101 \mathrm{MHz}\right.$, methanol- $\left.d_{4}\right) \delta 173.2$, 155.8, 145.2, 97.7, 82.5, 75.9, 73.5, 72.1, 62.0, 56.0, 28.7, 23.7, 22.4, 22.0. HRMS (ESI-TOF) $m / z:[\mathrm{M}+\mathrm{H}]^{+}$and $[\mathrm{M}+\mathrm{Na}]^{+}$Calcd for $\mathrm{C}_{16} \mathrm{H}_{30} \mathrm{~N}_{3} \mathrm{O}_{7} 376.2078$ and $\mathrm{C}_{16} \mathrm{H}_{29} \mathrm{~N}_{3} \mathrm{O}_{7} \mathrm{Na}$ 398.1898; found 376.2075 and 398.1898 .

\section{ASSOCIATED CONTENT}

\section{Supporting Information}

The Supporting Information is available free of charge on the ACS Publications website at DOI: 10.1021/acs.joc.8b01949.

Associated analytical data $\left({ }^{1} \mathrm{H} \mathrm{NMR},{ }^{13} \mathrm{C}\left\{{ }^{1} \mathrm{H}\right\}\right.$ NMR for all compounds (PDF)

Crystallographic data for 4 (CIF)

Crystallographic data for $\mathbf{2 4}$ (CIF)

\section{AUTHOR INFORMATION}

\section{Corresponding Authors}

*E-mail: m.d.witte@rug.nl.

*E-mail: a.j.minnaard@rug.nl.

ORCID ${ }^{\circ}$

Martin D. Witte: 0000-0003-4660-2974

Adriaan J. Minnaard: 0000-0002-5966-1300

Notes

The authors declare no competing financial interest.

\section{ACKNOWLEDGMENTS}

We acknowledge the China Scholarship Council for supporting J. Zhang and the Dutch Science Foundation NWO, grant no. 022.004.027 to N. Eisink. Prof. Dr. E. Otten and J. van der Velde, BSc, are acknowledged for X-ray crystallography.

\section{REFERENCES}

(1) Elshahawi, S. I.; Shaaban, K. A.; Kharel, M. K.; Thorson, J. S. A comprehensive review of glycosylated bacterial natural products. Chem. Soc. Rev. 2015, 44, 7591-7697.

(2) (a) Brimacombe, S. J. The Synthesis of Rare Sugars. Angew. Chem., Int. Ed. Engl. 1969, 8, 401-409. (b) Skarbek, K.; Milewska, M. J. Biosynthetic and synthetic access to amino sugars. Carbohydr. Res. 2016, 434, 44-71.

(3) Martínez, R. F.; Liu, Z.; Glawar, A. F.; Yoshihara, A.; Izumori, K.; Fleet, G. W. J.; Jenkinson, S. F. Short and Sweet: d-Glucose to LGlucose and L-Glucuronic Acid. Angew. Chem., Int. Ed. 2014, 53, $1160-1162$. 
(4) Lenagh-Snow, G. M. J.; Araújo, N.; Jenkinson, S. F.; Martínez, R. F.; Shimada, Y.; Yu, C.-Y.; Kato, A.; Fleet, G. W. J. Azetidine Iminosugars from the Cyclization of 3,5-Di-O-triflates of $\alpha$-Furanosides and of 2,4-Di-O-triflates of $\beta$-Pyranosides Derived from Glucose. Org. Lett. 2012, 14, 2142-2145.

(5) Lenagh-Snow, G. M. J.; Jenkinson, S. F.; Newberry, S. J.; Kato, A.; Nakagawa, S.; Adachi, I.; Wormald, M. R.; Yoshihara, A.; Morimoto, K.; Akimitsu, K.; Izumori, K.; Fleet, G. W. J. Eight Stereoisomers of Homonojirimycin from d-Mannose. Org. Lett. 2012, 14, 2050-2053.

(6) (a) Song, W.; Cai, J.; Zou, X.; Wang, X.; Hu, J.; Yin, J. Applications of controlled inversion strategies in carbohydrate synthesis. Chin. Chem. Lett. 2018, 29, 27-34. (b) Hanessian, S.; Massé, R.; Nakagawa, T. Aminoglycoside antibiotics: Studies directed toward the selective modification of hydroxyl groups: Synthesis of 3'epiparomamine and 3'-epineamine. Can. J. Chem. 1978, 56, 15091517. (c) Shinozaki, K.; Mizuno, K.; Masaki, Y. Syntheses of Optically Active, Unusual, and Biologically Important Hydroxy-Amino Acids from D-Glucosamine. Heterocycles 1996, 43, 11-14. (d) Emmerson, D. P. G.; Villard, R.; Mugnaini, C.; Batsanov, A.; Howard, J. A. K.; Hems, W. P.; Tooze, R. P.; Davis, B. G. Precise structure activity relationships in asymmetric catalysis using carbohydrate scaffolds to allow ready fine tuning: dialkylzinc-aldehyde additions. Org. Biomol. Chem. 2003, 1, 3826-3838.

(7) Conway, R.; Nagel, J.; Stick, R.; Tilbrook, D. Further Aspects of the Reduction of Dithiocarbonates with Tributyltin Hydride and Deuteride. Aust. J. Chem. 1985, 38, 939-945.

(8) Miyake, T.; Tsuchiya, T.; Takahashi, Y.; Umezawa, S. Reaction of methyl 2-deoxy-3-O-sulfonyl-2-p-toluenesulfonamido- $\alpha$-and $\beta$-dglucopyranoside derivatives with halide ions. Carbohydr. Res. 1981, $89,255-269$.

(9) Lin, T.-H.; Kováč, P.; Glaudemans, C. P. J. Improved synthesis of the 2-, 3-, and 4-deoxy derivatives from methyl $\beta$-d-galactopyranoside. Carbohydr. Res. 1989, 188, 228-238.

(10) Huang, G. L.; Dai, Y. P. Solid-Phase Synthesis of Allosamidin. Synlett 2010, 2010, 1554-1556.

(11) Mori, T.; Ichiyanagi, T.; Kondo, H.; Tokunaga, T.; Oda, T.; Munakata, T. Studies on new antibiotic lividomycins. J. Antibiot. 1971, 24, 339-346.

(12) Miyashita, M.; Chida, N.; Yoshikohsi, A. Synthesis of the precursor of (+)-thienamycin utilizing d-glucosamine. J. Chem. Soc., Chem. Commun. 1982, 1354-1356.

(13) McEvoy, F. J.; Weiss, M. J.; Baker, B. R. The Synthesis of 9-(2Amino-2-deoxy- $\beta$-d-allopyranosyl)-6-dimethylaminopurine, an Ana$\log$ of the Aminonucleoside Derived from Puromycin. J. Am. Chem. Soc. 1960, 82, 205-209.

(14) Kandula, M. Compositions and Methods for the Treatment of Inflammation. U.S. Patent 20150148306 A1, May 28, 2015.

(15) (a) Jäger, M.; Minnaard, A. J. Regioselective modification of unprotected glycosides. Chem. Commun. 2016, 52, 656-664. (b) Dimakos, V.; Garrett, G. E.; Taylor, M. S. Site-Selective, Copper-Mediated O-Arylation of Carbohydrate Derivatives. J. Am. Chem. Soc. 2017, 139, 15515-15521. (c) Wei, X.-F.; Shimizu, Y.; Kanai, M. An Expeditious Synthesis of Sialic Acid Derivatives by Copper (I)-Catalyzed Stereodivergent Propargylation of Unprotected Aldoses. ACS Cent. Sci. 2016, 2, 21-26.

(16) Jäger, M.; Hartmann, M.; de Vries, J. G.; Minnaard, A. J. Catalytic regioselective oxidation of glycosides. Angew. Chem., Int. Ed. 2013, 52, 7809-12.

(17) Armenise, N.; Tahiri, N.; Eisink, N. N. H. M.; Denis, M.; Jäger, M.; De Vries, J. G.; Witte, M. D.; Minnaard, A. J. Deuteration enhances catalyst lifetime in palladium-catalysed alcohol oxidation. Chem. Commun. 2016, 52, 2189-2191.

(18) Eisink, N. N. H. M.; Lohse, J.; Witte, M. D.; Minnaard, A. J. Regioselective oxidation of unprotected 1,4 linked glucans. Org. Biomol. Chem. 2016, 14, 4859-4864.

(19) Jumde, V. R.; Eisink, N. N. H. M.; Witte, M. D.; Minnaard, A. J. C3 Epimerization of Glucose, via Regioselective Oxidation and Reduction. J. Org. Chem. 2016, 81, 11439-11443.
(20) Eisink, N. N. H. M.; Witte, M. D.; Minnaard, A. J. Regioselective Carbohydrate Oxidations: A Nuclear Magnetic Resonance (NMR) Study on Selectivity, Rate, and Side-Product Formation. ACS Catal. 2017, 7, 1438-1445.

(21) Chung, K.; Waymouth, R. M. Selective Catalytic Oxidation of Unprotected Carbohydrates. ACS Catal. 2016, 6, 4653-4659.

(22) Jeanloz, R. W. The Synthesis of d-Allosamine Hydrochloride. J. Am. Chem. Soc. 1957, 79, 2591-2592.

(23) Jäger, V.; Schröter, D. Synthesis of Amino Sugars via Isoxazolines: D-Allosamine. Synthesis 1990, 1990, 556-560.

(24) Wong, C. H.; Ichikawa, Y.; Krach, T.; Gautheron-Le Narvor, C.; Dumas, D. P.; Look, G. C. Probing the acceptor specificity of $\beta$ 1,4-galactosyltransferase for the development of enzymatic synthesis of novel oligosaccharides. J. Am. Chem. Soc. 1991, 113, 8137-8145.

(25) Cai, L.; Guan, W.; Kitaoka, M.; Shen, J.; Xia, C.; Chen, W.; Wang, P. G. A chemoenzymatic route to $N$-acetylglucosamine-1phosphate analogues: substrate specificity investigations of $N$ acetylhexosamine 1-kinase. Chem. Commun. 2009, 2944-2946.

(26) Zhan, Z.-L.; Ren, F.-X.; Zhao, Y.-M. Facile synthesis of dlividosamine. Carbohydr. Res. 2010, 345, 315-317.

(27) For other syntheses of lividosamine see: (a) Hasegawa, A.; Tanahashi, E.; Kiso, M. Some reactions of a furanoid 2-aminoglycal derivative. Carbohydr. Res. 1980, 79, 255-264. (b) Arita, H.; Fukukawa, K.; Matsushima, Y. Studies on Amino-hexoses. XVI. Synthesis of Deoxy-analogues of N-Acetyl-d-glucosamine. Bull. Chem. Soc. Jpn. 1972, 45, 3614-3619. (c) Ravindran, B.; Deshpande, S. G.; Pathak, T. Vinylsulfone-modified carbohydrates: first general route to d-lividosamine (2-amino-2,3-dideoxy-d-glucose) and its new analogues. Tetrahedron 2001, 57, 1093-1098. (d) de Guchteneere, E.; Fattori, D.; Vogel, P. Total asymmetric syntheses of d-lividosamine and 2-acetamido-2,3-dideoxy-d-arabino-hexose derivatives. Tetrahedron 1992, 48, 10603-10620. (e) Jegou, E.; Cleophax, J.; Leboul, J.; Gero, S. D. A facile synthesis of derivatives of lividosamine, a component of lividomycin B. Carbohydr. Res. 1975, 45, 323-326. (f) zu Reckendorf, W. M.; Bonner, W. A. Sulphur substitution compounds of aminosugars. V. The synthesis of 2-amino-2,3-dideoxy3-mercapto-d-allose and-d-glucose derivatives by displacement reactions. Tetrahedron 1963, 19, 1711-1720.

(28) Nair, V.; Sinhababu, A. K. Selective Transformations of Sugar Tosyl Hydrazones to Deoxy and Unsaturated Sugars. J. Org. Chem. 1978, 43, 5013-5017.

(29) Schröder, S. P.; Wu, L.; Artola, M.; Hansen, T.; Offen, W. A.; Ferraz, M. J.; Li, K.-Y.; Aerts, J. M. F. G.; van der Marel, G. A.; Codée, J. D. C.; Davies, G. J.; Overkleeft, H. S. Gluco- $1 H$-imidazole: A New Class of Azole-Type $\beta$-Glucosidase Inhibitor. J. Am. Chem. Soc. 2018, $140,5045-5048$.

(30) (a) Richter, C.; Nguyen Trung, M.; Mahrwald, R. Multicomponent Cascade Reactions of Unprotected Ketoses and Amino Acids - Access to a Defined Configured Quaternary Stereogenic Center. J. Org. Chem. 2015, 80, 10849-10865. (b) Richter, C.; Krumrey, M.; Bahri, M.; Trunschke, S.; Mahrwald, R. AmineCatalyzed Cascade Reactions of Unprotected Aldoses-An Operationally Simple Access to Defined Configured Stereotetrads or Stereopentads. ACS Catal. 2016, 6, 5549-5552. (c) Richter, C.; Krumrey, M.; Klaue, K.; Mahrwald, R. Cascade Reactions of Unprotected Ketoses with Ketones - A Stereoselective Access to C-Glycosides. Eur. J. Org. Chem. 2016, 2016, 5309-5320.

(31) Wan, I. C.; Witte, M. D.; Minnaard, A. J. Site-selective carboncarbon bond formation in unprotected monosaccharides using photoredox catalysis. Chem. Commun. 2017, 53, 4926-4929.

(32) Qian, X.; Sujino, K.; Otter, A.; Palcic, M. M.; Hindsgaul, O. Chemoenzymatic Synthesis of $\alpha$-(1 $\rightarrow 3)$-Gal(NAc)-Terminating Glycosides of Complex Tertiary Sugar Alcohols. J. Am. Chem. Soc. 1999, 121, 12063-12072.

(33) Nicolaou, K. C.; Li, Y.; Fylaktakidou, K. C.; Mitchell, H. J.; Wei, H.; Weyershausen, B. Total Synthesis of Apoptolidin: Part 1. Retrosynthetic Analysis and Construction of Building Blocks. Angew. Chem., Int. Ed. 2001, 40, 3849-3854. 
(34) Carpenter, J.; Northrup, A.; Chung, D.; Wiener, J.; Kim, S.; MacMillan, D. Total Synthesis and Structural Revision of Callipeltoside C. Angew. Chem., Int. Ed. 2008, 47, 3568-3572.

(35) Conley, N. R.; Labios, L. A.; Pearson, D. M.; McCrory, C. C. L.; Waymouth, R. M. Aerobic Alcohol Oxidation with Cationic Palladium Complexes: Insights into Catalyst Design and Decomposition. Organometallics 2007, 26, 5447-5453.

(36) Schöberl, C.; Jäger, V. 3- and 4-Uloses Derived from N-AcetylD-glucosamine: A Unique Pair of Complementary Organocatalysts for Asymmetric Epoxidation of Alkenes. Adv. Synth. Catal. 2012, 354, 790-796.

(37) Lemieux, R. U.; Gunner, S. W. Hydrogenation of alkyl 2oximino- $\alpha$-D-arabino-hexopyranosides. Can. J. Chem. 1968, 46, 397400.

(38) Sakuda, S.; Isogai, A.; Makita, T.; Matsumoto, S.; Koseki, K.; Kodama, H.; Suzuki, A. Structures of Allosamidins, Novel Insect Chitinase Inhibitors, Produced by Actinomycetes. Agric. Biol. Chem. 1987, 51, 3251-3259.

(39) Dmitriev, B. A.; Knirel, Y. A.; Kochetkov, N. K. Selective cleavage of glycosidic linkages: Studies with the model compound benzyl 2 -acetamido-2-deoxy-3-O- $\beta$-D-galactopyranosyl- $\alpha$-D-glucopyranoside. Carbohydr. Res. 1973, 29, 451-457.

(40) Okumura, H.; Azuma, I.; Kiso, M.; Hasegawa, A. The equilibrium compositions and conformations of some carbohydrate analogs of $\mathrm{N}$-acetylmuramoyl-l-alanyl-d-isoglutamine as determined by ${ }^{1}$ H-n.m.r. spectroscopy. Carbohydr. Res. 1983, 117, 298-303. 